\title{
Modeling population dynamics of roseate terns (Sterna dougallii) in the Northwest Atlantic Ocean
}

\author{
Manuel García-Quismondo ${ }^{\mathrm{a}, \mathrm{b}, \mathrm{c}, *}$, Ian C.T. Nisbet $^{\mathrm{d}}$, Carolyn Mostello $^{\mathrm{e}}$, J. Michael Reed ${ }^{\mathrm{b}}$ \\ ${ }^{a}$ Research Group on Natural Computing, University of Sevilla, ETS Ingeniería Informática, Av. Reina Mercedes, s/n, Sevilla 41012, Spain \\ ${ }^{\mathrm{b}}$ Dept. of Biology, Tufts University, Medford, MA 02155, USA \\ ' Darrin Fresh Water Institute, Rensselaer Polytechnic Institute, 110 8th Street, 307 MRC, Troy, NY 12180, USA \\ ' I.C.T. Nisbet E' Company, 150 Alder Lane, North Falmouth, MA 02556, USA \\ e Massachusetts Division of Fisheries E Wildlife, 1 Rabbit Hill Road, Westborough, MA 01581, USA
}

\section{A R T I C L E I N F O}

\section{Article history:}

Received 12 September 2017

Received in revised form 5 December 2017

Accepted 6 December 2017

\section{Keywords:}

Roseate terns

Metapopulation

Source-sink

Population persistence

Islands

\begin{abstract}
A B S T R A C T
The endangered population of roseate terns (Sterna dougallii) in the Northwestern Atlantic Ocean consists of a network of large and small breeding colonies on islands. This type of fragmented population poses an exceptional opportunity to investigate dispersal, a mechanism that is fundamental in population dynamics and is crucial to understand the spatio-temporal and genetic structure of animal populations. Dispersal is difficult to study because it requires concurrent data compilation at multiple sites. Models of population dynamics in birds that focus on dispersal and include a large number of breeding sites are rare in literature. In this work, we propose a stochastic simulation model that captures the dispersal dynamics of this population of roseate terns. The colonization and decolonization (abandonment) of breeding colonies are modeled as discrete events that follow different dynamics than dispersal. We show that our model reproduces the properties of this population that have been observed in field data. We also analyzed the sensitivity of our model to alterations in different variables, and study the impact of these alterations in the model dynamics. Our results suggest that large colony population size exhibits a threshold sensitivity to adult survival, and that regional persistence is maintained by the larger populations.
\end{abstract}

(c) 2017 Elsevier B.V. All rights reserved.

\section{Introduction}

Understanding the dynamics of populations is central to assessments of extinction risk, invasion, and community dynamics, as well as other topics (Rhodes et al., 1996). Dispersal is an important mechanism in population dynamics, and is fundamental to understanding metapopulation structure, source-sink dynamics, population synchrony, gene flow and genetic structure, as well as colonization, range shifts and species conservation (e.g. Hanski, 2001; Saura et al., 2014; Whitlock, 2001). However, dispersal is a difficult demographic parameter to study, especially in continuous habitats, because it requires detecting individuals at multiple locations (e.g. Jønsson et al., 2016; Koenig et al., 1996). Dispersal can be somewhat easier to study in discontinuous populations, such as those formed by colonial seabirds nesting on islands, but it still requires data collection at multiple sites, and large samples at each site may be required to estimate rates and dynamics of dispersal if

\footnotetext{
* Corresponding author at: Research Group on Natural Computing, University of Sevilla, ETS Ingeniería Informática, Av. Reina Mercedes, s/n, Sevilla 41012, Spain.
}

these are small (Crespin et al., 2006; Dolman, 2012; Opdam, 1991; Serrano et al., 2005).

Comprehensive models of populations in fragmented landscapes are uncommon for bird populations, particularly those distributed among a large number of breeding sites. Here, we present a population dynamics model for the population of roseate terns (Sterna dougallii; Aves, Laridae) that breeds on islands distributed along about $300 \mathrm{~km}$ of the Atlantic coast of the USA south and west of Cape Cod, Massachusetts. Although roseate terns have nested on 32 islands in recent decades, only 5-10 have been occupied in any one year (see Fig. 1, Tables 1 and 2). A few of the islands are occupied every year by large populations, while other islands contain small populations that persist for a while, disappear, and are later recolonized (Tables 1 and 2). This fits Dolman's categorization of a mainland-island metapopulation (where the 'mainland' in this case is the set of islands with large populations), and of a fragmented population having regional population effects (Dolman, 2012); for convenience, we will refer to our system as being a metapopulation (Harrison and Taylor, 1997; Stith et al., 1996). This metapopulation of terns has been studied intensively since 1988 (e.g. Nisbet et al., 2016; Spendelow et al., 2016), with 
Table 1

Site coordinates.

\begin{tabular}{|c|c|c|}
\hline Colony site & Latitude & Longitude \\
\hline Bird Island, Marion, MA & $41^{\circ} 40^{\prime} \mathrm{N}$ & $70^{\circ} 43^{\prime} \mathrm{W}$ \\
\hline Dead Neck-Sampsons Island, MA & $41^{\circ} 37^{\prime} \mathrm{N}$ & $70^{\circ} 25^{\prime} \mathrm{W}$ \\
\hline Ram Island, Mattapoisett, MA & $41^{\circ} 37^{\prime} \mathrm{N}$ & $70^{\circ} 48^{\prime} \mathrm{W}$ \\
\hline Muskeget sandbars, Nantucket, MA & $41^{\circ} 34^{\prime} \mathrm{N}$ & $70^{\circ} 30^{\prime} \mathrm{W}$ \\
\hline Muskeget Island, Nantucket, MA & $41^{\circ} 34^{\prime} \mathrm{N}$ & $70^{\circ} 31^{\prime} \mathrm{W}$ \\
\hline Penikese Island, Gosnold, MA & $41^{\circ} 27^{\prime} \mathrm{N}$ & $70^{\circ} 56^{\prime} \mathrm{W}$ \\
\hline Nashawena Island, Gosnold, MA & $41^{\circ} 26^{\prime} \mathrm{N}$ & $70^{\circ} 52^{\prime} \mathrm{W}$ \\
\hline Little Beach, MV, MA & $41^{\circ} 24^{\prime} \mathrm{N}$ & $70^{\circ} 30^{\prime} \mathrm{W}$ \\
\hline Haystack Point, MV, MA & $41^{\circ} 24^{\prime} \mathrm{N}$ & $70^{\circ} 32^{\prime} \mathrm{W}$ \\
\hline Norton Point, MV, MA & $41^{\circ} 21^{\prime} \mathrm{N}$ & $70^{\circ} 29^{\prime} \mathrm{W}$ \\
\hline Menemsha Pond, MV, MA & $41^{\circ} 20^{\prime} \mathrm{N}$ & $70^{\circ} 46^{\prime} \mathrm{W}$ \\
\hline Shore Rock, Ocean Point., CT & $41^{\circ} 18^{\prime} \mathrm{N}$ & $72^{\circ} 06^{\prime} \mathrm{W}$ \\
\hline Smith Point, Nantucket, MA & $41^{\circ} 17^{\prime} \mathrm{N}$ & $70^{\circ} 13^{\prime} \mathrm{W}$ \\
\hline Tuxis Island, CT & $41^{\circ} 16^{\prime} \mathrm{N}$ & $72^{\circ} 36^{\prime} \mathrm{W}$ \\
\hline Duck Island, CT & $41^{\circ} 15^{\prime} \mathrm{N}$ & $72^{\circ} 28^{\prime} \mathrm{W}$ \\
\hline Falkner Island, CT & $41^{\circ} 13^{\prime} \mathrm{N}$ & $72^{\circ} 39^{\prime} \mathrm{W}$ \\
\hline Great Gull Island, NY & $41^{\circ} 12^{\prime} \mathrm{N}$ & $72^{\circ} 07^{\prime} \mathrm{W}$ \\
\hline Gardiners Point Island (Fort Tyler), NY & $41^{\circ} 08^{\prime} \mathrm{N}$ & $72^{\circ} 09^{\prime} \mathrm{W}$ \\
\hline Gardiners Island, NY & $41^{\circ} 06^{\prime} \mathrm{N}$ & $72^{\circ} 07^{\prime} \mathrm{W}$ \\
\hline Hicks Island, East Hampton, NY & $41^{\circ} 01^{\prime} \mathrm{N}$ & $72^{\circ} 04^{\prime} \mathrm{W}$ \\
\hline Gardiners Island-Cartwright Point, NY & $41^{\circ} 01^{\prime} \mathrm{N}$ & $72^{\circ} 06^{\prime} \mathrm{W}$ \\
\hline Young's Island, Smithtown, NY & $40^{\circ} 55^{\prime} \mathrm{N}$ & $73^{\circ} 11^{\prime} \mathrm{W}$ \\
\hline Warner Island, Southampton, NY & $40^{\circ} 51^{\prime} \mathrm{N}$ & $72^{\circ} 30^{\prime} \mathrm{W}$ \\
\hline Lanes Island, Southampton, NY & $40^{\circ} 50^{\prime} \mathrm{N}$ & $72^{\circ} 31^{\prime} \mathrm{W}$ \\
\hline Greater Greenbacks Island, Southampton, NY & $40^{\circ} 50^{\prime} \mathrm{N}$ & $72^{\circ} 32^{\prime} \mathrm{W}$ \\
\hline East Inlet Island, Brookhaven, NY & $40^{\circ} 47^{\prime} \mathrm{N}$ & $72^{\circ} 45^{\prime} \mathrm{W}$ \\
\hline Pattersquash Island, Brookhaven, NY & $40^{\circ} 45^{\prime} \mathrm{N}$ & $73^{\circ} 50^{\prime} \mathrm{W}$ \\
\hline Sexton Island, Islip, NY & $40^{\circ} 39^{\prime} \mathrm{N}$ & $73^{\circ} 14^{\prime} \mathrm{W}$ \\
\hline Cedar Beach, Babylon, NY & $40^{\circ} 38^{\prime} \mathrm{N}$ & $73^{\circ} 20^{\prime} \mathrm{W}$ \\
\hline Goose Flat, Babylon, NY & $40^{\circ} 38^{\prime} \mathrm{N}$ & $73^{\circ} 23^{\prime} \mathrm{W}$ \\
\hline West End Jones Beach, Hempstead, NY & $40^{\circ} 35^{\prime} \mathrm{N}$ & $73^{\circ} 33^{\prime} \mathrm{W}$ \\
\hline Breezy Point, Queens City, NY & $40^{\circ} 33^{\prime} \mathrm{N}$ & $73^{\circ} 57^{\prime} \mathrm{W}$ \\
\hline
\end{tabular}

some historical information dating back to 1870 (see Supplementary Material). It is thought to be demographically isolated from other metapopulations of the species in the North Atlantic region, with negligible rates of interchange of individuals (U.S. Fish and Wildlife Service, 2010). One of the challenging aspects of modeling its demography is that there exists a time lag of 3-4 years between the time chicks hatch, and the time they start breeding (Lebreton et al., 2003). Monitoring and Capture-Mark-Recapture (CMR) studies have yielded estimates of numbers of breeding pairs, breeding productivity, adult and juvenile survival and dispersal rates, and ages at first breeding, for all the large colonies and most of the medium-sized colonies in this metapopulation for all or part of the 28-year period (see Supplementary Material, Tables 2-10). However, estimates of demographic parameters are incomplete for some of the medium-sized colonies and for all the small colonies; the small colonies have been occupied transitorily, with frequent colonizations and decolonizations.

We developed a model based on stochastic estimates of the missing demographic parameters that aims to reproduce the observed statistical behavior of the actual metapopulation. Our model is based on assumptions about the spatio-temporal dynamics that describe the allocation of dispersing individuals (adults that do not return to the same site in successive years, and juveniles that do not return to their natal site when they recruit to the breeding population) among the breeding colonies within the system. Our goal was to construct a model that conforms to the following observed properties of the metapopulation: (1) total numbers remain within the range of $3000-8000$ pairs over long periods of time ( $>50$ years); (2) about $90 \%$ of the total numbers breed in $2-4$ large colonies (500-2000 pairs), which remain large for periods of 20-50 years; ( 3 ) small colonies ( $\leq 10$ pairs) are occupied and abandoned intermittently, and the majority of them do not reach medium nor large size; (4) the frequency of formation of a new large colony is about once every 30 years; (5) at about the same fre- quency, one new medium-sized colony becomes established and persists for a long period ( $>30$ years). A model that reproduces these features of the wild population could be used realistically for population viability analyses and for assessing potential effects of perturbations (e.g., climate change, predator introductions) and management actions.

\section{Methods}

To capture the dynamics of this system, we propose a population model consisting of a set of finite difference equations (FDEs). Our model reproduces the fecundity, survival and dispersal of a population distributed among $M$ sites over $T$ years. In this discrete-time model, each time step $t, 1 \leq t \leq T$, represents a year in the system, and each site is denoted by an index $i, 1 \leq i \leq M$. The number of breeding pairs in site $i$ and year $t$ is represented as $N_{i, t}$. Before describing the model equations, the following definitions are introduced:

Definition 1. We define the annual proportion $a_{i, t}$ of birds at site $i$ at year $t 1 \leq i \leq M, 1 \leq t \leq T$ as the fraction of the total population in year $t$ that breed at site $i$ in that year: $a_{i, t}=N_{i, t} / \sum_{j=1}^{M} N_{j, t}$. We consider that adults that bred at site $i$ in year $t$, and juveniles that were raised at the same site in year $t-4$, are 'attached' to site $i$. The time lag for returning juveniles is driven by the species' biology, where hatch-year birds migrate, stay in the non-breeding grounds for 1.5 years, visit breeding sites at age 2 years, and breed for the first time at ages 3 or 4 years (Lebreton et al., 2003; Nisbet, 2014). Our model ignores birds that breed at age 3 because these usually breed late in the season and raise few young (Nisbet, 2014). We also assume that 'unattached' birds, defined as those that emigrated from other breeding sites, are attracted to site $i$ in proportion to the number of breeding pairs at site $i$ relative to proportions at other sites.

Definition 2. We define the intrinsic quality $q_{i}$ of site $i$ as the average annual proportion of the population found at this site over all years. Intrinsic quality is calculated as $q_{i}=$ $\sum_{t=k_{1}}^{k_{T_{\max }}} a_{i, k_{t}} /\left(\sum_{j=1}^{M} \sum_{l=k_{1}}^{k_{T_{\max }}} a_{j, k_{l}}\right)$, where $k_{1}, \ldots, k_{T_{\max }}, k_{1} \leq k_{T_{\max }}$ is the population size across the range of years for which historical data on the number of pairs on each site are available. In our case, this range spans from 1988 to 2015, i.e., $k_{1}=1988$ and $k_{T_{\max }}=2015$.

Definition 3. We define a 'small' site as one for which the maximum number of pairs found in the historical data is 10 (see Supplementary Material, Appendix S1). We define that a 'small' site $i$ in year $t$ is in colonization mode $\left(\operatorname{col}_{i, t}=1\right)$ if it can receive immigrants from other sites in year $t+1$ and its productivity in the following year $t+1\left(H_{i, t+1}\right.$, see below) can be greater than 0 . Likewise, we define that a small site is in decolonization mode $\left(\right.$ decol $\left._{i, t}=1\right)$ if it is not in colonization mode and cannot enter colonization mode in year $t+1$. It is important to note that a site cannot be simultaneously in colonization and decolonization modes, i.e., it is not possible that $\operatorname{col}_{i, t}=1 \wedge$ decol $_{i, t}=1$, for any $i, t \mid 1 \leq i \leq M, 1 \leq t \leq T$. However, it is possible that a site can be simultaneously in neither of these modes. As an example, a site may not receive immigrants nor produce juveniles at a given year $t$ but may be eligible for colonization in year $t+1$. Large sites are always considered to be in colonization mode.

Both annual proportion and quality values were calculated from a dataset of nest counts at each site from 1988 to 2015 (see Supplementary Material, Table 2). We consider that $\operatorname{col}_{i, 1}=0$ and $\operatorname{decol}_{i, 1}=0,1 \leq i \leq M$ for small sites. In other words, in the initial state of the model, all small colonies are not in colonization mode but are susceptible to enter this mode from year 2 and on. 
Table 2

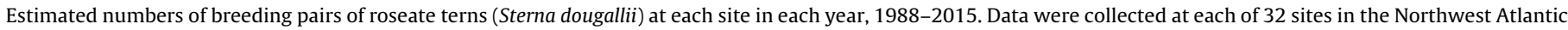

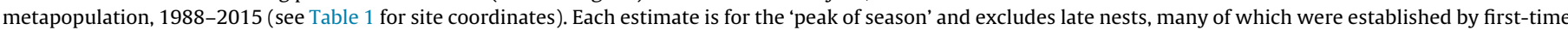
breeders.

\begin{tabular}{|c|c|c|c|c|c|}
\hline Colony site & 1988 & 1989 & 1990 & 1991 & 1992 \\
\hline Bird Island, Marion, MA & 1572 & 1473 & 1547 & 1728 & 1375 \\
\hline Dead Neck-Sampsons Island, MA & 13 & 27 & 0 & 0 & 0 \\
\hline Ram Island, Mattapoisett, MA & 0 & 0 & 0 & 0 & 0 \\
\hline Muskeget sandbars, Nantucket, MA & 0 & 0 & 0 & 0 & 0 \\
\hline Muskeget Island, Nantucket, MA & 0 & 0 & 0 & 0 & 0 \\
\hline Penikese Island, Gosnold, MA & 0 & 0 & 0 & 0 & 0 \\
\hline Nashawena Island, Gosnold, MA & 3 & 0 & 3 & 0 & 4 \\
\hline Little Beach, MV, MA & 0 & 0 & 0 & 0 & 0 \\
\hline Haystack Point, MV, MA & 0 & 4 & 0 & 0 & 0 \\
\hline Norton Point, MV, MA & 0 & 0 & 0 & 0 & 0 \\
\hline Menemsha Pond, MV, MA & 0 & 0 & 0 & 2 & 0 \\
\hline Shore Rock, Ocean Point., CT & 0 & 1 & 0 & 0 & 0 \\
\hline Smith Point, Nantucket, MA & 0 & 0 & 0 & 0 & 0 \\
\hline Tuxis Island, CT & 0 & 4 & 0 & 0 & 0 \\
\hline Duck Island, CT & 2 & 5 & 0 & 0 & 0 \\
\hline Falkner Island, CT & 157 & 96 & 150 & 149 & 107 \\
\hline Great Gull Island, NY & 1004 & 960 & 1026 & 1204 & 964 \\
\hline Gardiners Point Island (Fort Tyler), NY & 0 & 0 & 0 & 0 & 0 \\
\hline Gardiners Island, NY & 0 & 0 & 0 & 0 & 0 \\
\hline Hicks Island, East Hampton, NY & 0 & 0 & 2 & 4 & 4 \\
\hline Gardiners Island-Cartwright Point, NY & 0 & 0 & 0 & 0 & 0 \\
\hline Young's Island, Smithtown, NY & 0 & 0 & 0 & 0 & 0 \\
\hline Warner Island, Southampton, NY & 0 & 6 & 17 & 40 & 39 \\
\hline Lanes Island, Southampton, NY & 0 & 0 & 0 & 6 & 16 \\
\hline Greater Greenbacks Island, Southampton, NY & 0 & 2 & 0 & 0 & 0 \\
\hline East Inlet Island, Brookhaven, NY & 2 & 40 & 20 & 6 & 1 \\
\hline Pattersquash Island, Brookhaven, NY & 0 & 0 & 0 & 0 & 0 \\
\hline Sexton Island, Islip, NY & 0 & 0 & 0 & 0 & 0 \\
\hline Cedar Beach, Babylon, NY & 93 & 66 & 94 & 120 & 80 \\
\hline Goose Flat, Babylon, NY & 0 & 0 & 0 & 0 & 0 \\
\hline West End Jones Beach, Hempstead, NY & 13 & 2 & 0 & 0 & 0 \\
\hline Breezy Point, Queens City, NY & 0 & 0 & 0 & 0 & 0 \\
\hline Colony site & 1993 & 1994 & 1995 & 1996 & 1997 \\
\hline Bird Island, Marion, MA & 1319 & 1238 & 1250 & 996 & 1179 \\
\hline Dead Neck-Sampsons Island, MA & 0 & 0 & 0 & 0 & 0 \\
\hline Ram Island, Mattapoisett, MA & 0 & 76 & 197 & 719 & 253 \\
\hline Muskeget sandbars, Nantucket, MA & 0 & 0 & 0 & 0 & 0 \\
\hline Muskeget Island, Nantucket, MA & 0 & 0 & 0 & 0 & 0 \\
\hline Penikese Island, Gosnold, MA & 0 & 0 & 0 & 0 & 0 \\
\hline Nashawena Island, Gosnold, MA & 0 & 0 & 0 & 0 & 0 \\
\hline Little Beach, MV, MA & 0 & 0 & 0 & 0 & 0 \\
\hline Haystack Point, MV, MA & 0 & 0 & 0 & 0 & 0 \\
\hline Norton Point, MV, MA & 0 & 0 & 0 & 0 & 0 \\
\hline Menemsha Pond, MV, MA & 0 & 0 & 0 & 0 & 0 \\
\hline Shore Rock, Ocean Point., CT & 0 & 0 & 0 & 0 & 0 \\
\hline Smith Point, Nantucket, MA & 0 & 0 & 0 & 0 & 0 \\
\hline Tuxis Island, CT & 0 & 1 & 0 & 0 & 0 \\
\hline Duck Island, CT & 0 & 0 & 0 & 0 & 0 \\
\hline Falkner Island, CT & 130 & 123 & 125 & 135 & 136 \\
\hline Great Gull Island, NY & 1040 & 1138 & 1056 & 1064 & 1455 \\
\hline Gardiners Point Island (Fort Tyler), NY & 0 & 0 & 157 & 4 & 23 \\
\hline Gardiners Island, NY & 0 & 0 & 0 & 0 & 0 \\
\hline Hicks Island, East Hampton, NY & 0 & 0 & 0 & 0 & 0 \\
\hline Gardiners Island-Cartwright Point, NY & 4 & 14 & 0 & 1 & 5 \\
\hline Young's Island, Smithtown, NY & 0 & 0 & 0 & 0 & 0 \\
\hline Warner Island, Southampton, NY & 28 & 53 & 34 & 24 & 41 \\
\hline Lanes Island, Southampton, NY & 12 & 35 & 59 & 36 & 16 \\
\hline Greater Greenbacks Island, Southampton, NY & 0 & 0 & 0 & 0 & 2 \\
\hline East Inlet Island, Brookhaven, NY & 12 & 0 & 0 & 0 & 0 \\
\hline Pattersquash Island, Brookhaven, NY & 0 & 0 & 0 & 0 & 0 \\
\hline Sexton Island, Islip, NY & 0 & 0 & 0 & 0 & 0 \\
\hline Cedar Beach, Babylon, NY & 51 & 58 & 37 & 0 & 0 \\
\hline Goose Flat, Babylon, NY & 0 & 0 & 0 & 0 & 9 \\
\hline West End Jones Beach, Hempstead, NY & 0 & 0 & 0 & 0 & 0 \\
\hline Breezy Point, Queens City, NY & 2 & 2 & 2 & 2 & 4 \\
\hline Colony site & 1998 & 1999 & 2000 & 2001 & 2002 \\
\hline Bird Island, Marion, MA & 1113 & 1148 & 1130 & 1062 & 505 \\
\hline Dead Neck-Sampsons Island, MA & 0 & 0 & 0 & 0 & 0 \\
\hline Ram Island, Mattapoisett, MA & 543 & 630 & 988 & 626 & 952 \\
\hline Muskeget sandbars, Nantucket, MA & 0 & 0 & 0 & 0 & 0 \\
\hline Muskeget Island, Nantucket, MA & 0 & 5 & 0 & 0 & 0 \\
\hline Penikese Island, Gosnold, MA & 0 & 0 & 0 & 0 & 0 \\
\hline Nashawena Island, Gosnold, MA & 0 & 0 & 0 & 0 & 0 \\
\hline
\end{tabular}


Table 2 (Continued)

\begin{tabular}{|c|c|c|c|c|c|}
\hline Colony site & 1988 & 1989 & 1990 & 1991 & 1992 \\
\hline Little Beach, MV, MA & 0 & 0 & 0 & 0 & 0 \\
\hline Haystack Point, MV, MA & 0 & 0 & 0 & 0 & 0 \\
\hline Norton Point, MV, MA & 0 & 0 & 0 & 0 & 0 \\
\hline Menemsha Pond, MV, MA & 0 & 0 & 0 & 0 & 0 \\
\hline Shore Rock, Ocean Point., CT & 0 & 0 & 0 & 0 & 0 \\
\hline Smith Point, Nantucket, MA & 0 & 0 & 0 & 0 & 0 \\
\hline Tuxis Island, CT & 0 & 0 & 0 & 0 & 0 \\
\hline Duck Island, CT & 0 & 0 & 0 & 0 & 0 \\
\hline Falkner Island, CT & 115 & 110 & 110 & 95 & 65 \\
\hline Great Gull Island, NY & 1690 & 1747 & 1762 & 1562 & 1505 \\
\hline Gardiners Point Island (Fort Tyler), NY & 0 & 0 & 0 & 0 & 0 \\
\hline Gardiners Island, NY & 46 & 2 & 0 & 0 & 0 \\
\hline Hicks Island, East Hampton, NY & 0 & 0 & 0 & 0 & 0 \\
\hline Gardiners Island-Cartwright Point, NY & 0 & 2 & 0.00 & 6 & 156 \\
\hline Young's Island, Smithtown, NY & 0 & 0 & 0 & 0 & 0 \\
\hline Warner Island, Southampton, NY & 31 & 28 & 9 & 0 & 0 \\
\hline Lanes Island, Southampton, NY & 32 & 14 & 18 & 5 & 0 \\
\hline Greater Greenbacks Island, Southampton, NY & 2 & 0 & 0 & 4 & 1 \\
\hline East Inlet Island, Brookhaven, NY & 0 & 0 & 0 & 0 & 0 \\
\hline Pattersquash Island, Brookhaven, NY & 0 & 0 & 0 & 0 & 0 \\
\hline Sexton Island, Islip, NY & 0 & 0 & 0 & 0 & 0 \\
\hline Cedar Beach, Babylon, NY & 0 & 0 & 0 & 0 & 0 \\
\hline Goose Flat, Babylon, NY & 16 & 1 & 2 & 4 & 1 \\
\hline West End Jones Beach, Hempstead, NY & 0 & 0 & 0 & 0 & 0 \\
\hline Breezy Point, Queens City, NY & 5 & 0 & 0 & 0 & 0 \\
\hline Colony site & 2003 & 2004 & 2005 & 2006 & 2007 \\
\hline Bird Island, Marion, MA & 904 & 554 & 680 & 1111 & 919 \\
\hline Dead Neck-Sampsons Island, MA & 0 & 0 & 0 & 0 & 0 \\
\hline Ram Island, Mattapoisett, MA & 557 & 936 & 724 & 463 & 661 \\
\hline Muskeget sandbars, Nantucket, MA & 0 & 0 & 0 & 0 & 0 \\
\hline Muskeget Island, Nantucket, MA & 0 & 0 & 0 & 0 & 0 \\
\hline Penikese Island, Gosnold, MA & 251 & 9 & 76 & 48 & 102 \\
\hline Nashawena Island, Gosnold, MA & 0 & 0 & 0 & 0 & 0 \\
\hline Little Beach, MV, MA & 0 & 0 & 0 & 0 & 0 \\
\hline Haystack Point, MV, MA & 0 & 0 & 0 & 0 & 0 \\
\hline Norton Point, MV, MA & 0 & 0 & 0 & 0 & 0 \\
\hline Menemsha Pond, MV, MA & 0 & 0 & 0 & 0 & 0 \\
\hline Shore Rock, Ocean Point., CT & 0 & 0 & 0 & 0 & 0 \\
\hline Smith Point, Nantucket, MA & 0 & 0 & 0 & 0 & 0 \\
\hline Tuxis Island, CT & 0 & 0 & 0 & 0 & 0 \\
\hline Duck Island, CT & 0 & 0 & 0 & 0 & 0 \\
\hline Falkner Island, CT & 45 & 37 & 44 & 62 & 54 \\
\hline Great Gull Island, NY & 1613 & 1352 & 1195 & 1227 & 1546 \\
\hline Gardiners Point Island (Fort Tyler), NY & 2 & 0 & 0 & 0 & 0 \\
\hline Gardiners Island, NY & 0 & 0 & 0 & 0 & 0 \\
\hline Hicks Island, East Hampton, NY & 0 & nd & 0 & 0 & 0 \\
\hline Gardiners Island-Cartwright Point, NY & 155 & 248 & 90 & 80 & 216 \\
\hline Young's Island, Smithtown, NY & 1 & 0 & 0 & 0 & 0 \\
\hline Warner Island, Southampton, NY & 1 & 1 & 0 & 0 & 0 \\
\hline Lanes Island, Southampton, NY & 4 & 4 & 2 & 0 & 2 \\
\hline Greater Greenbacks Island, Southampton, NY & 0 & 0 & 0 & 2 & 2 \\
\hline East Inlet Island, Brookhaven, NY & 0 & 0 & 0 & 0 & 0 \\
\hline Pattersquash Island, Brookhaven, NY & 0 & 0 & 2 & 0 & 0 \\
\hline Sexton Island, Islip, NY & 2 & 2 & 2 & 0 & 0 \\
\hline Cedar Beach, Babylon, NY & 0 & 0 & 0 & 0 & 0 \\
\hline Goose Flat, Babylon, NY & 0 & 11 & 11 & 25 & 2 \\
\hline West End Jones Beach, Hempstead, NY & 0 & 0 & 0 & 0 & 0 \\
\hline Breezy Point, Queens City, NY & 0 & 0 & 0 & 0 & 0 \\
\hline Colony site & 2008 & 2009 & 2010 & 2011 & 2012 \\
\hline Bird Island, Marion, MA & 747 & 708 & 735 & 937 & 814 \\
\hline Dead Neck-Sampsons Island, MA & 0 & 0 & 0 & 0 & 0 \\
\hline Ram Island, Mattapoisett, MA & 566 & 588 & 584 & 377 & 439 \\
\hline Muskeget sandbars, Nantucket, MA & 0 & 0 & 0 & 0 & 0 \\
\hline Muskeget Island, Nantucket, MA & 0 & 0 & 0 & 0 & 0 \\
\hline Penikese Island, Gosnold, MA & 66 & 43 & 37 & 34 & 9 \\
\hline Nashawena Island, Gosnold, MA & 0 & 0 & 0 & 0 & 0 \\
\hline Little Beach, MV, MA & 0 & 0 & 0 & 0 & 0 \\
\hline Haystack Point, MV, MA & 0 & 0 & 0 & 0 & 0 \\
\hline Norton Point, MV, MA & 0 & 50 & 36 & 0 & 5 \\
\hline Menemsha Pond, MV, MA & 0 & 0 & 0 & 0 & 0 \\
\hline Shore Rock, Ocean Point., CT & 0 & 0 & 0 & 0 & 0 \\
\hline Smith Point, Nantucket, MA & 0 & 0 & 0 & 0 & 0 \\
\hline Tuxis Island, CT & 0 & 0 & 0 & 0 & 0 \\
\hline Duck Island, CT & 0 & 0 & 0 & 0 & 0 \\
\hline Falkner Island, CT & 32 & 28 & 45 & 48 & 36 \\
\hline Great Gull Island, NY & 1288 & 1413 & 1303 & 1439 & 1596 \\
\hline
\end{tabular}


Table 2 (Continued)

\begin{tabular}{|c|c|c|c|c|c|}
\hline Colony site & 1988 & 1989 & 1990 & 1991 & 1992 \\
\hline Gardiners Point Island (Fort Tyler), NY & 0 & 0 & 0 & 0 & 0 \\
\hline Gardiners Island, NY & 0 & 0 & 0 & 0 & 0 \\
\hline Hicks Island, East Hampton, NY & 0 & 0 & 0 & 0 & 0 \\
\hline Gardiners Island-Cartwright Point, NY & 27 & 27 & 0 & 0 & 0 \\
\hline Young's Island, Smithtown, NY & 0 & 0 & 0 & 0 & 0 \\
\hline Warner Island, Southampton, NY & 0 & 0 & 0 & 0 & 0 \\
\hline Lanes Island, Southampton, NY & 0 & 0 & 0 & 0 & 0 \\
\hline Greater Greenbacks Island, Southampton, NY & 2 & 2 & 0 & 0 & 0 \\
\hline East Inlet Island, Brookhaven, NY & 0 & 0 & 0 & 0 & 0 \\
\hline Pattersquash Island, Brookhaven, NY & 0 & 0 & 0 & 0 & 0 \\
\hline Sexton Island, Islip, NY & 0 & 0 & 0 & 0 & 0 \\
\hline Cedar Beach, Babylon, NY & 0 & 0 & 0 & 0 & 0 \\
\hline Goose Flat, Babylon, NY & 1 & 0 & 0 & 0 & 0 \\
\hline West End Jones Beach, Hempstead, NY & 0 & 0 & 0 & 0 & 0 \\
\hline Breezy Point, Queens City, NY & 0 & 0 & 0 & 0 & 0 \\
\hline Colony site & 2013 & 2014 & 2015 & & \\
\hline Bird Island, Marion, MA & 772 & 1121 & 1127 & & \\
\hline Dead Neck-Sampsons Island, MA & 0 & 0 & 0 & & \\
\hline Ram Island, Mattapoisett, MA & 535 & 682 & 735 & & \\
\hline Muskeget sandbars, Nantucket, MA & 0 & 0 & 0 & & \\
\hline Muskeget Island, Nantucket, MA & 0 & 0 & 0 & & \\
\hline Penikese Island, Gosnold, MA & 0 & 20 & 23 & & \\
\hline Nashawena Island, Gosnold, MA & 0 & 0 & 0 & & \\
\hline Little Beach, MV, MA & 0 & 0 & 7 & & \\
\hline Haystack Point, MV, MA & 0 & 0 & 0 & & \\
\hline Norton Point, MV, MA & 35 & 0 & 35 & & \\
\hline Menemsha Pond, MV, MA & 0 & 0 & 0 & & \\
\hline Shore Rock, Ocean Point., CT & 0 & 0 & 0 & & \\
\hline Smith Point, Nantucket, MA & 0 & 0 & 0 & & \\
\hline Tuxis Island, CT & 0 & 0 & 0 & & \\
\hline Duck Island, CT & 0 & 0 & 0 & & \\
\hline Falkner Island, CT & 26 & 21 & 36 & & \\
\hline Great Gull Island, NY & 1543 & 1459 & 1659 & & \\
\hline Gardiners Point Island (Fort Tyler), NY & 0 & 0 & 0 & & \\
\hline Gardiners Island, NY & 0 & 0 & 0 & & \\
\hline Hicks Island, East Hampton, NY & 0 & 0 & 0 & & \\
\hline Gardiners Island-Cartwright Point, NY & 0 & 0 & 0 & & \\
\hline Young's Island, Smithtown, NY & 0 & 0 & 0 & & \\
\hline Warner Island, Southampton, NY & 0 & 0 & & & \\
\hline Lanes Island, Southampton, NY & 0 & 0 & 0 & & \\
\hline Greater Greenbacks Island, Southampton, NY & 0 & 0 & 0 & & \\
\hline East Inlet Island, Brookhaven, NY & 0 & 0 & 0 & & \\
\hline Pattersquash Island, Brookhaven, NY & 0 & 0 & 0 & & \\
\hline Sexton Island, Islip, NY & 0 & 0 & 0 & & \\
\hline Cedar Beach, Babylon, NY & 0 & 0 & 0 & & \\
\hline Goose Flat, Babylon, NY & 0 & 0 & 0 & & \\
\hline West End Jones Beach, Hempstead, NY & 0 & 0 & 0 & & \\
\hline Breezy Point, Queens City, NY & 0 & 0 & 0 & & \\
\hline
\end{tabular}

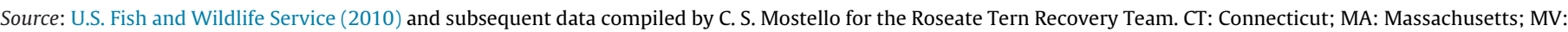
Martha's Vineyard; NY: New York (Long Island).

The model equations are defined as follows: If no colonization or decolonization event occurs on site $i$ and year $t$, then $N_{i, t+1}=\operatorname{round}\left(\left(A S_{i, t}+J S_{i, t}+I_{i, t+1}\right)\right)$, where round is the standard round function for real numbers; i.e., rounding to the nearest integer. The terms in this equation are defined as follows:

- $A S_{i, t}$ (for Adults Staying) represents the number of adult pairs that breed at site $i$ in year $t$ and return to breed at the same site in year $t+1$. $A S_{i, t}$ is calculated as $A S_{i, t}=\omega_{i, t} \times \phi_{i, t} \times N_{i, t}$, where $\omega_{i, t}$ is the proportion of adult birds that survive between consecutive years, and $\phi_{i, t}$ is the site fidelity ratio; that is, the proportion of pairs that breed at site $i$ in year $t$ and return to the same site in year $t+1$, conditional to survival in year $t . \omega_{i, t}$ was estimated using a log-linear regression model $\omega_{i, t}=1 /\left(1+\exp \left(\omega^{0}+\omega^{1} \times q_{i}+\omega^{2} \times a_{i, t}\right)\right)+\epsilon^{\omega_{i, t}}$, where $\epsilon^{\omega_{i, t}}$ is the model error for site $i$ and year $t$. The ground-truth values for $\omega_{i, t}$ are shown in Table 6 . The model coefficients are $\omega^{0}=1.53, \omega^{1}=9.368 \times 10^{-5}$ and $\omega^{2}=-3.161 \times 10^{-1}$. Values for $\phi_{i, t}$ are estimated using the log-linear regression model $\phi_{i, t}=1 /\left(1+\exp \left(\phi^{0}+\phi^{1} \times q_{i}+\phi^{2} \times a_{i, t}\right)\right)+\epsilon^{\phi_{i, t}}$. The groundtruth values for $\phi_{i, t}$ were obtained from the frequencies of same-site transitions shown in Fig. 2 in Spendelow et al. (2016), and the model coefficients are $\phi^{0}=0.931, \phi^{1}=3.818$ and $\phi^{2}=-0.741$.

- $J S_{i, t}$ (for Juveniles Staying) represents the number of juvenile birds (counted as pairs) raised to fledging at site $i$ in year $t-3$ that survive to enter the breeding population and breed in site $i$ in year $t+1$. We assume that all surviving juveniles enter the breeding population at age 4 (see caption to Table 8 ). $J S_{i, t}$ is calculated as $J S_{i, t}=\operatorname{col}_{i, t} \times H_{i, t-3} \times \gamma_{i, t-3} \times \rho_{i, t} \times N_{i, t-3} / 2$, where:

- $H_{i, t-3}$ represents the productivity as the mean number of chicks reared to fledging per pair at site $i$ in year $t-3$. We used the log-linear regression model: $H_{i, t}=\max H /\left(1+\exp \left(H^{0}+H^{1} \times N_{i, t}+H^{2} \times q_{i}\right)\right)+\epsilon^{H_{i, t}}$ to estimate productivity values. The ground-truth values for $H_{i, t}$ were derived from a dataset on average productivity at most sites in years 1998 to 2015 (Table 3 ). $\operatorname{maxH}=1.82$ is the maximum productivity value from this dataset, and is a scaling factor for the log-linear model. Based on empirical data and using log-linear regression, the model coefficients were estimated to be $H^{0}=-0.214, H^{1}=0$ and $H^{2}=-0.006$. 
Table 3

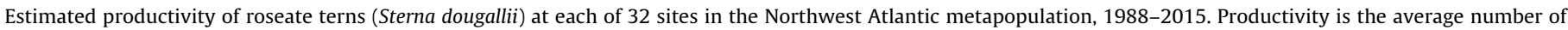

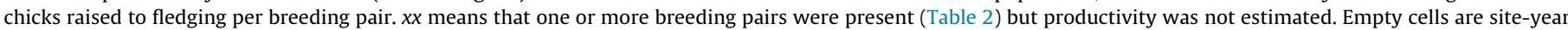

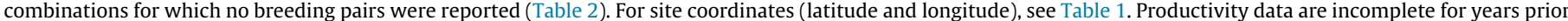
to 1998.

\begin{tabular}{|c|c|c|c|c|c|}
\hline Colony site & 1998 & 1999 & 2000 & 2001 & 2002 \\
\hline Bird Island, Marion, MA & 1.40 & 1.40 & 1.10 & 1.00 & 1.02 \\
\hline Ram Island, Mattapoisett, MA & 1.45 & 1.04 & 1.11 & 1.05 & 0.96 \\
\hline Muskeget sandbars, Nantucket, MA & & $\mathrm{xx}$ & & & \\
\hline \multicolumn{6}{|l|}{ Penikese Island, Gosnold, MA } \\
\hline \multicolumn{6}{|l|}{ Nashawena Island, Gosnold, MA } \\
\hline \multicolumn{6}{|l|}{ Haystack Point, MV, MA } \\
\hline \multicolumn{6}{|l|}{ Norton Point, MV, MA } \\
\hline Falkner Island, CT & 0.74 & 0.70 & 0.75 & 0.72 & 0.18 \\
\hline Great Gull Island, NY & 1.50 & 1.40 & 1.27 & 1.13 & 0.90 \\
\hline \multicolumn{6}{|l|}{ Gardiners Point Island (Fort Tyler), NY } \\
\hline Gardiners Island, NY & $\mathrm{xx}$ & 1.00 & & & \\
\hline Gardiners Island-Cartwright Point, NY & & $\mathrm{xx}$ & & 0.75 & $\mathrm{xx}$ \\
\hline \multicolumn{6}{|l|}{ Young's Island, Smithtown, NY } \\
\hline Warner Island, Southampton, NY & 1.05 & 1.17 & 0.00 & & \\
\hline Lanes Island, Southampton, NY & $\mathrm{xx}$ & 0.00 & 0.00 & 0.00 & \\
\hline Greater Greenbacks Island, Southampton, NY & $\mathrm{xx}$ & & & $\mathrm{xx}$ & $\mathrm{xx}$ \\
\hline \multicolumn{6}{|l|}{ Pattersquash Island, Brookhaven, NY } \\
\hline \multicolumn{6}{|l|}{ Sexton Island, Islip, NY } \\
\hline Goose Flat, Babylon, NY & $\mathrm{xx}$ & $\mathrm{xx}$ & $\mathrm{xx}$ & $\mathrm{xx}$ & $\mathrm{xx}$ \\
\hline Breezy Point, Queens City, NY & $\mathrm{xx}$ & & & & \\
\hline Colony site & 2003 & 2004 & 2005 & 2006 & 2007 \\
\hline Bird Island, Marion, MA & 1.25 & 1.25 & 0.95 & 1.29 & 1.26 \\
\hline Ram Island, Mattapoisett, MA & 1.14 & 0.92 & 0.93 & 1.00 & 1.16 \\
\hline \multicolumn{6}{|l|}{ Muskeget sandbars, Nantucket, MA } \\
\hline Penikese Island, Gosnold, MA & 0.87 & 0.97 & 0.79 & 0.44 & 1.54 \\
\hline \multicolumn{6}{|l|}{ Nashawena Island, Gosnold, MA } \\
\hline \multicolumn{6}{|l|}{ Haystack Point, MV, MA } \\
\hline Norton Point, MV, MA & & & & & \\
\hline Falkner Island, CT & 0.26 & 0.53 & 0.67 & 0.34 & 0.54 \\
\hline Great Gull Island, NY & 1.00 & 1.17 & 1.60 & 1.30 & 0.90 \\
\hline Gardiners Point Island (Fort Tyler), NY & $\mathrm{xx}$ & & & & \\
\hline Gardiners Island, NY & & & & & \\
\hline Gardiners Island-Cartwright Point, NY & $\mathrm{xx}$ & $\mathrm{xx}$ & $\mathrm{xx}$ & $\mathrm{xx}$ & $\mathrm{xx}$ \\
\hline Young's Island, Smithtown, NY & 0.00 & & & & \\
\hline Warner Island, Southampton, NY & 0.00 & $\mathrm{xx}$ & & & \\
\hline Lanes Island, Southampton, NY & $\mathrm{xx}$ & $\mathrm{xx}$ & $\mathrm{xx}$ & & $\mathrm{xx}$ \\
\hline Greater Greenbacks Island, Southampton, NY & & & & $\mathrm{xx}$ & $\mathrm{xx}$ \\
\hline Pattersquash Island, Brookhaven, NY & & & $\mathrm{xx}$ & & \\
\hline Sexton Island, Islip, NY & $\mathrm{xx}$ & $\mathrm{xx}$ & $\mathrm{xx}$ & & \\
\hline Goose Flat, Babylon, NY & & $\mathrm{xx}$ & 1.82 & $\mathrm{xx}$ & $\mathrm{xx}$ \\
\hline Breezy Point, Queens City, NY & & & & & \\
\hline Colony site & 2008 & 2009 & 2010 & 2011 & 2012 \\
\hline Bird Island, Marion, MA & 1.12 & 1.44 & 1.33 & 1.23 & 1.12 \\
\hline Ram Island, Mattapoisett, MA & 1.25 & 1.04 & 1.30 & 1.10 & 1.31 \\
\hline Muskeget sandbars, Nantucket, MA & & & & & \\
\hline Penikese Island, Gosnold, MA & 1.42 & 0.73 & $\mathrm{xx}$ & $\mathrm{xx}$ & 0.11 \\
\hline Nashawena Island, Gosnold, MA & & 0.00 & & & \\
\hline Haystack Point, MV, MA & & & & & \\
\hline Norton Point, MV, MA & & $\mathrm{xx}$ & 0.20 & & 1.30 \\
\hline Falkner Island, CT & 1.06 & 1.15 & 0.98 & 0.91 & 0.87 \\
\hline Great Gull Island, NY & 0.98 & 1.10 & 1.40 & 1.30 & 0.80 \\
\hline Gardiners Point Island (Fort Tyler), NY & & & & & \\
\hline Gardiners Island, NY & & & & & \\
\hline Gardiners Island-Cartwright Point, NY & $\mathrm{xx}$ & $\mathrm{xx}$ & & & \\
\hline Young's Island, Smithtown, NY & & & & & \\
\hline Warner Island, Southampton, NY & & & & & \\
\hline Lanes Island, Southampton, NY & & & & & \\
\hline Greater Greenbacks Island, Southampton, NY & $\mathrm{xx}$ & $\mathrm{xx}$ & & & \\
\hline Pattersquash Island, Brookhaven, NY & & & & & \\
\hline Sexton Island, Islip, NY & & & & & \\
\hline Goose Flat, Babylon, NY & $\mathrm{xx}$ & & & & \\
\hline Breezy Point, Queens City, NY & & & & & \\
\hline Colony site & 2013 & 2014 & 2015 & & \\
\hline Bird Island, Marion, MA & 1.34 & 0.85 & 0.86 & & \\
\hline Ram Island, Mattapoisett, MA & 1.31 & 0.98 & 0.87 & & \\
\hline Muskeget sandbars, Nantucket, MA & & & & & \\
\hline Penikese Island, Gosnold, MA & & 1.08 & 1.12 & & \\
\hline Nashawena Island, Gosnold, MA & & & & & \\
\hline Haystack Point, MV, MA & & & 0.00 & & \\
\hline Norton Point, MV, MA & 1.20 & & $\mathrm{xx}$ & & \\
\hline
\end{tabular}


Table 3 (Continued)

\begin{tabular}{|c|c|c|c|c|c|}
\hline Colony site & 1998 & 1999 & 2000 & 2001 & 2002 \\
\hline Falkner Island, CT & 1.00 & 0.64 & 1.02 & & \\
\hline Great Gull Island, NY & 1.16 & 1.00 & 1.25 & & \\
\hline \multicolumn{6}{|c|}{ Gardiners Point Island (Fort Tyler), NY } \\
\hline \multicolumn{6}{|c|}{ Gardiners Island, NY } \\
\hline \multicolumn{6}{|c|}{ Gardiners Island-Cartwright Point, NY } \\
\hline \multicolumn{6}{|c|}{ Young's Island, Smithtown, NY } \\
\hline \multicolumn{6}{|l|}{ Warner Island, Southampton, NY } \\
\hline \multicolumn{6}{|c|}{ Lanes Island, Southampton, NY } \\
\hline \multicolumn{6}{|c|}{ Greater Greenbacks Island, Southampton, NY } \\
\hline \multicolumn{6}{|c|}{ Pattersquash Island, Brookhaven, NY } \\
\hline \multicolumn{6}{|c|}{ Sexton Island, Islip, NY } \\
\hline \multicolumn{6}{|l|}{ Goose Flat, Babylon, NY } \\
\hline Breezy Point, Queens City, NY & & & & & \\
\hline
\end{tabular}

Source: U.S. Fish and Wildlife Service (2010) and subsequent data compiled by C. S. Mostello for the Roseate Tern Recovery Team. MV: Martha's Vineyard.

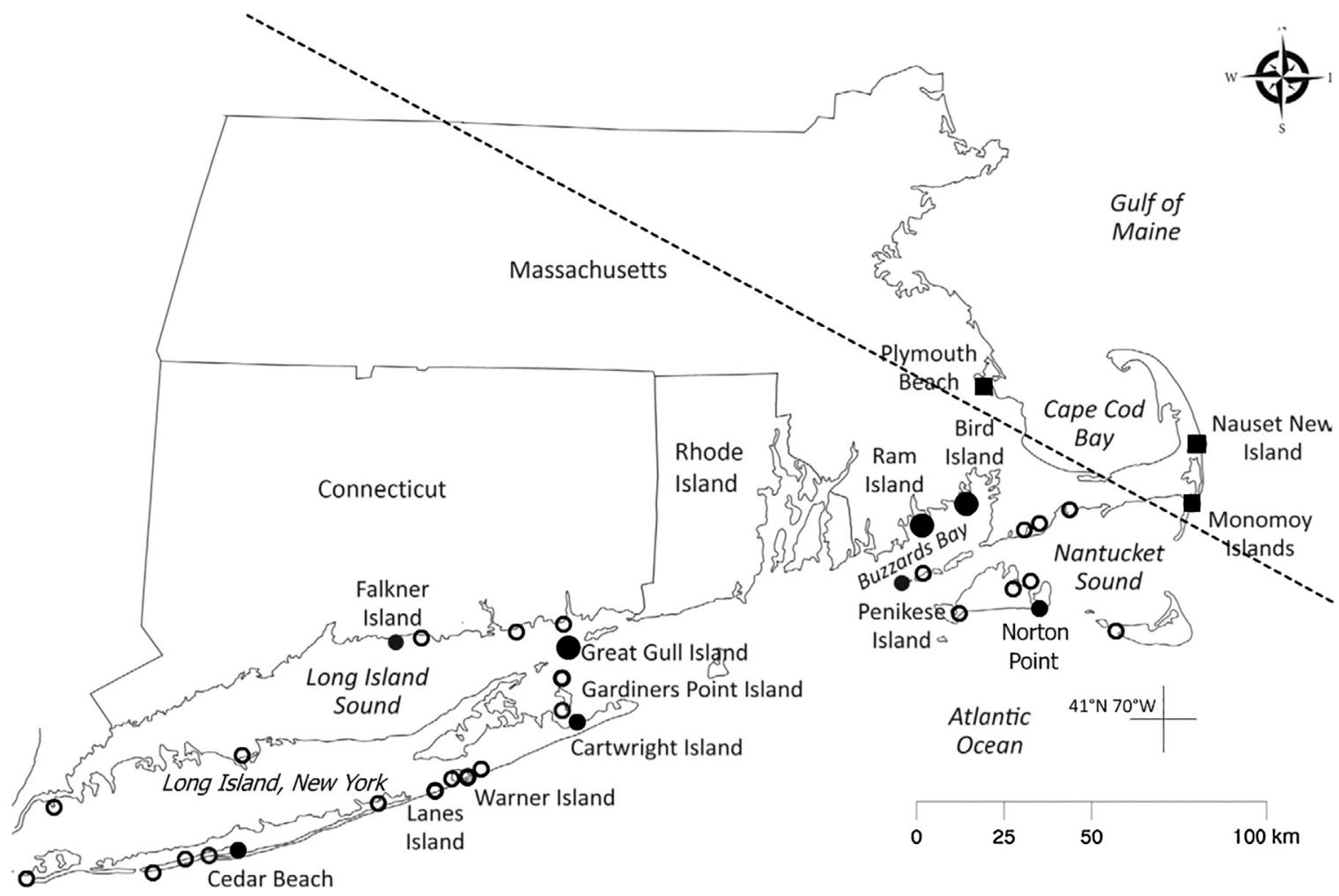

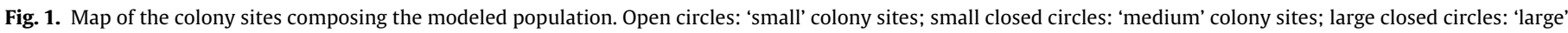
colony sites. The dashed line marks the boundary of the modeled population; the colonies to the right of this line are not included in our model.

- $\gamma_{i, t-3}$ represents the survival rate of juveniles raised at site $i$ in year $t-3$ to year $t+1 . \gamma_{i, t-3}$ was estimated using a loglinear regression model $\gamma_{i, t-3}=\gamma_{w} \times \omega_{i, t}^{2} /\left(1+\exp \left(\gamma^{0}+\gamma^{1} \times\right.\right.$ $\left.\left.q_{i}\right)\right)+\epsilon^{\gamma_{i, t}}$. The ground-truth values for $\gamma_{i, t}$ are shown in Table 7 , and the model coefficients were adjusted as $\gamma^{0}=-0.510$ and $\gamma^{1}=2.56$. This estimated value was multiplied by $\omega_{i, t}^{2}$ to yield survival to age 4 . This value is multiplied by a scaling factor $\gamma_{w}$ empirically adjusted to 1.218 , that minimizes the difference between the number of pairs in large sites at the end of the model simulation and in the historical data (Table 2).

- $\rho_{i, t}$ represents juvenile philopatry; that is, the proportion of birds that were reared at site $i$ in year $t-3$ and first bred at site $i$ in year $t+1$, conditional on survival to age 4 . $\rho_{i, t}$ is calculated as $\rho_{i, t}=\phi_{i, t} \times \eta_{i}$, where $\eta_{i}$ represents the ratio of juvenile fidelity divided by adult fidelity in site $i$ and year $t$. We estimated these values using the log-linear regression model $\eta_{i}=1 /\left(1+\exp \left(\eta^{0}+\eta^{1} \times N_{i, t}\right)\right)+\epsilon \eta_{\mathrm{i}}$. The ground-truth values for $\eta_{i}$ were obtained by dividing the values in the diagonals of
Tables 9 and 10, and the model coefficients were adjusted as $\eta^{0}=0.444$ and $\eta^{1}=0.002$.

The equations describing $J S_{i, t}$ and $J L_{i, t}$ (see below) include a factor of $1 / 2$ to convert from individual juveniles $\left(H_{i, t-3}\right)$ to pairs of adults; this was done for calculational convenience.

- $A L_{i, t}$ (for Adults Leaving) represents the number of pairs of adult birds that bred at site $i$ in year $t$, survived to year $t+1$ but did not breed at site $i$ in year $t+1 . A L_{i, t}$ is calculated as $A L_{i, t}=\left(1-\phi_{i, t}\right) \times \omega_{i, t} \times N_{i, t}$.

- $J L_{i, t}$ (for Juveniles Leaving) represents the number of pairs of juvenile birds that were raised to fledging at site $i$ in year $t-3$, survived to breed for the first time in year $t$, but did not breed at site $i$ in year $t+1$. $J L_{i, t}$ is calculated as $J L_{i, t}=H_{i, t-3} \times \gamma_{i, t} \times\left(1-\rho_{i, t}\right) \times N_{i, t-3} / 2$.

- $I_{i, t}$ for (Immigrants) represents the pairs that breed at site $i$ in year $t+1$ that did not breed at site $i$ in year $t . I_{i, t}$ is calculated as follows. First, a pool with the total number of pairs leaving their site and available for immigration into all sites is calculated as pool $l_{t}=$ 
Table 4

Changes in numbers of breeding pairs at colonies within the WWG, 1988-2015.

\begin{tabular}{|c|c|c|c|c|c|c|c|c|}
\hline \multirow{2}{*}{ Pairs in year $t$} & & \multicolumn{7}{|c|}{ Pairs in year $t+1$} \\
\hline & & 0 & 1 & 2 & 3 & 4 & 5 & $6-10$ \\
\hline 0 & & 541 & 4 & 11 & 1 & 6 & 2 & 2 \\
\hline 1 & & 7 & 1 & 1 & & & 1 & \\
\hline 2 & & 12 & 1 & 9 & & 3 & 1 & \\
\hline 3 & & 2 & & & & & & \\
\hline 4 & & 6 & 2 & 1 & 2 & & 1 & \\
\hline 5 & & 5 & & & & & & \\
\hline $6-10$ & & 2 & 1 & & & & & \\
\hline $11-20$ & & 2 & 1 & 1 & & 1 & 1 & 1 \\
\hline $\begin{array}{l}21-50 \\
51-100\end{array}$ & & 6 & & 2 & & & & 2 \\
\hline $101-300$ & & & & & & 1 & & 1 \\
\hline $300-800$ & & & & & & & & \\
\hline $801-1200$ & & & & & & & & \\
\hline $1200-1800$ & & & & & & & & \\
\hline Pairs in year $t$ & $11-20$ & $21-50$ & $51-100$ & $101-300$ & $300-800$ & $801-1200$ & 1200 & -1800 \\
\hline 0 & 2 & 3 & 2 & 2 & & & & \\
\hline 1 & 1 & & & & & & & \\
\hline 2 & & 1 & & & & & & \\
\hline 3 & & & & & & & & \\
\hline 4 & 1 & 1 & & & & & & \\
\hline 5 & & 1 & & & & & & \\
\hline 06-10 & 3 & & 1 & & & & & \\
\hline $11-20$ & 3 & 6 & & & & & & \\
\hline $21-50$ & 3 & 19 & 3 & 1 & & & & \\
\hline $51-100$ & & 7 & 7 & 5 & & & & \\
\hline $101-300$ & & 1 & 5 & 12 & 2 & & & \\
\hline $300-800$ & & & & & 15 & 7 & & \\
\hline $801-1200$ & & & & & 7 & 14 & 2 & \\
\hline $1200-1800$ & & & & & & 3 & 23 & \\
\hline
\end{tabular}

Source: Table 2.

Table 5

Probability of decolonization in relation to colony size.

\begin{tabular}{lll}
\hline Pairs in year $t$ & Number of cases & Probability of decolonization \\
\hline 1 & 11 & $64 \%$ \\
2 & 27 & $44 \%$ \\
3 & 2 & $100 \%$ \\
4 & 14 & $43 \%$ \\
5 & 6 & $83 \%$ \\
$6-10$ & 7 & $29 \%$ \\
$11-20$ & 15 & $13 \%$ \\
$21-50$ & 36 & $14 \%$ \\
$>50$ & 93 & $0 \%$ \\
\hline
\end{tabular}

Source: Table 2.

$\sum_{j=1}^{M}\left(A L_{j, t}+J L_{j, t}\right)-$ settlers $_{t}+$ desettlers $_{t}$. The terms settlers $t$ and desettlers $s_{t}$ refer to the sum of all pairs that colonize and decolonize any site in year $t$, and they are explained below.

We define a model $R I D_{i, t}$ for the relative distribution of this pool across the sites in year $t$ as a weighted sum of a deterministic component $I D_{i, t}$ and a stochastic component $I S_{i, t}$. The deterministic component is a log-linear regression model $I D_{i, t}=$ $1 /\left(1+\exp \left(I D^{0}+I D^{1} \times q_{i}+I D^{2} \times a_{i, t}\right)\right)+\epsilon_{i, t}^{I D}$.

The ground-truth values for $I D_{i, t}$ were calculated as $\left(\sum_{j=1, j \neq i}^{M} p_{j, i, t} \times N_{j, t}\right) /\left(\sum_{j=1, j \neq i}^{M} \sum_{k=1, k \neq j}^{M} p_{j, k, t} \times N_{j, t}\right)$, where values $p_{j, i, t}$ are the proportion of birds that leave site $j$ in year $t$ and breed in site $i$ in year $t+1$. These values are taken for transitions for adults between large sites from Table 4 in Spendelow et al. (2016) (Table 8) We assume that transition values for adults and juveniles are quantitatively similar. The model coefficients were adjusted as $I D^{0}=-0.485, I D^{1}=-6.025$ and $I D^{2}=2.117$.

The stochastic component $I S_{i, t}$ is drawn from a normal distribution $I S_{i, t} \sim \mathcal{N}\left(\mu, \sigma^{2}\right)$ with mean $(\mu=0.241)$ and standard deviation $(\sigma=0.176)$ derived from the ground-truth values for
Table 6

Site-specific estimates (mean \pm standard error) of annual survival of adult roseate terns during three time periods between 1988 and 2008. Survival of the 1991 cohort was severely reduced by a hurricane that passed through the staging area in August 1991

\begin{tabular}{llll}
\hline Site & \multicolumn{3}{l}{ Study period } \\
\cline { 2 - 4 } & $1988-1990$ and 1992-1999 & 1991 & $2000-2008$ \\
\hline Falkner Island & $0.863 \pm 0.010$ & $0.667 \pm 0.035$ & $0.805 \pm 0.019$ \\
Great Gull Island & $0.788 \pm 0.012$ & $0.807 \pm 0.067$ & $0.822 \pm 0.013$ \\
Bird Island & $0.851 \pm 0.007$ & $0.751 \pm 0.027$ & $0.830 \pm 0.007$ \\
Ram Island & $0.822 \pm 0.017$ & - & $0.841 \pm 0.008$ \\
Penikese Island & - & - & $0.771 \pm 0.028$ \\
\hline
\end{tabular}

Source: Spendelow et al. (2008) and Spendelow et al. (2016), Table 10 in Lebreton et al. (2003).

Table 7

Estimates of survival probability of juvenile roseate terns raised at the three major sites in the WWG from fledging to age 2 years over 10 years, 1988-1997. \pm denotes standard error. Survival of the 1991 cohort was severely reduced by a hurricane that passed through the staging area in August 1991.

\begin{tabular}{llll}
\hline Time period & \multicolumn{3}{l}{ Site } \\
\cline { 2 - 4 } & Falkner Island & Bird Island & Great Gull Island \\
\hline $1998-1990$ & $0.310 \pm 0.111$ & $0.325 \pm 0.087$ & $0.352 \pm 0.147$ \\
$1989-1991$ & $0.320 \pm 0.148$ & $0.340 \pm 0.090$ & $0.505 \pm 0.165$ \\
$1990-1992$ & $0.336 \pm 0.127$ & $0.378 \pm 0.088$ & $0.490 \pm 0.144$ \\
$1991-1993$ & $0.063 \pm 0.057$ & $0.185 \pm 0.095$ & $0.065 \pm 0.044$ \\
$1992-1994$ & $0.593 \pm 0.227$ & $0.748 \pm 0.204$ & $0.496 \pm 0.167$ \\
$1993-1995$ & $0.547 \pm 0.172$ & $0.275 \pm 0.109$ & $0.498 \pm 0.159$ \\
$1994-1996$ & $0.354 \pm 0.154$ & $0.415 \pm 0.150$ & $0.354 \pm 0.154$ \\
$1995-1997$ & $0.407 \pm 0.249$ & $0.046 \pm 0.066$ & $0.434 \pm 0.259$ \\
\hline
\end{tabular}

Source: Table 11 in Lebreton et al. (2003).

$I D_{i, t}$. The stochasticity of the model $r n$ is calculated as the average squared standard error of $I D_{i, t}$. We calculate this error as $r n=$ $\sqrt{\left(\sum_{j=1}^{M^{\prime}} \sum_{l=k_{1}}^{k_{T_{\max }}}\left(\text { epsilon }_{j, l}^{I D}\right)^{2}\right) /\left(M^{\prime} \times T_{\max }\right)}$, where, $j, 1 \leq j \leq M^{\prime}$ and $k_{1}, \ldots, k_{T_{\max }}$ represent the sites and years for which transition data across sites is available; in our case, large sites from 1988 through 2008.

The relative immigration distribution $R I D_{i, t}$ is defined as: $R I D_{i, t}=\left((1-r n) \times I D_{i, t}+r n \times I S_{i, t}\right) \times\left(\sum_{j=1}^{M} d_{i, j} \times N_{i, t}\right), \quad$ where $d_{i, j}, 1 \leq i, j \leq M$ is the distance in kilometers between sites $i$ and $j$. The component $\sum_{j=1}^{M} d_{i, j} \times N_{i, t}$ introduces a penalizing factor for sites with neighboring attractive breeding sites. This distribution is normalized, i.e., $R I D_{i, t}^{N o r m}=R I D_{i, t} /\left(\sum_{j=1}^{M} R I D_{j, t}\right)$. Finally, $I_{i, t}$ is calculated as $I_{i, t}=\operatorname{col}_{i, t} \times R I D_{i, t}^{N o r m} \times\left(\operatorname{pool}_{t}-A L_{i, t}-J L_{i, t}\right)$.

Non-large sites are subjected to colonization and decolonization events. The probabilities for colonization $P\left(\mathrm{Col}_{i}=1\right)$ and decolonization $P\left(\right.$ decol $\left._{i}=1\right)$ are site-specific, and are modeled as the probability for each site $i$ to transit from having 0 pairs in year $t$ $\left(N_{i, t}=0\right)$ to 1 or more pairs in year $t+1\left(N_{i, t+1}>0\right)$, and the probability to transit from $N_{i, t}>0$ to $N_{i, t}=0$, respectively. These are estimated as sample probabilities from Table 2 :

$P\left(\operatorname{col}_{i}=1\right)=\sum_{l=k_{1}}^{k_{T_{\max }}} \operatorname{Pred}\left(N_{i, l}=0\right) \times \operatorname{Pred}\left(N_{i, l+1}>0\right) /\left(\sum_{l=k_{1}}^{k_{T_{\max }}} \operatorname{Pred}\left(N_{i, l}=0\right)\right)$

and

$P\left(\operatorname{decol}_{i}=1\right)=\sum_{l=k_{1}}^{k_{T_{\max }}} \operatorname{Pred}\left(N_{i, l}>0\right) \times \operatorname{Pred}\left(N_{i, l+1}=0\right) /\left(\sum_{l=k_{1}}^{k_{T_{\max }}} \operatorname{Pred}\left(N_{i, l}>0\right)\right)$

where $k_{1}=1988$ and $k_{T_{\max }}=2015$ and $\operatorname{Pred}\left(N_{i, l}=0\right)$ and $\operatorname{Pred}\left(N_{i, l}>0\right) \mid 1 \leq i \leq M, l=k_{1} \leq l \leq k_{T_{\max }}$ are predicates that evaluate to 1 if $N_{i, l}=0$ and $N_{i, l}>0$, respectively, and evaluate to 0 otherwise. 
Table 8

Ages at first breeding of roseate terns at the three major colony sites in the WWG, 1988-1997.

\begin{tabular}{|c|c|c|c|c|}
\hline \multirow[t]{2}{*}{ Breeding site } & \multicolumn{4}{|l|}{ Age (years) } \\
\hline & 2 & 3 & 4 & 5 \\
\hline Falkner Island & $0.010 \pm 0.012$ & $0.515 \pm 0.085$ & $0.620 \pm 0.145$ & $0.912 \pm 0.244$ \\
\hline Bird Island & $0.046 \pm 0.036$ & $0.674 \pm 0.180$ & 1.000 & 1.000 \\
\hline Great Gull Island & $0.019 \pm 0.023$ & $0.448 \pm 0.159$ & $0.668 \pm 0.411$ & 1.000 \\
\hline
\end{tabular}

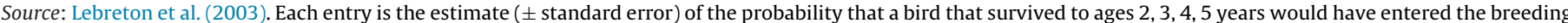

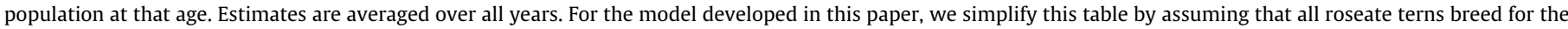

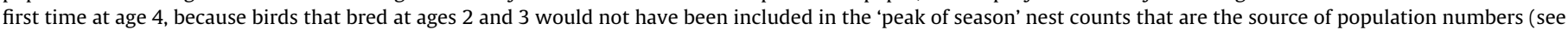
text of Supplementary Material).

Table 9

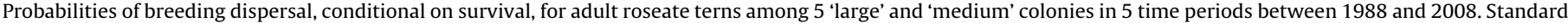
errors are in parentheses. - indicates that one or both sites were not occupied in the specified time periods.

\begin{tabular}{|c|c|c|c|c|c|}
\hline & \multicolumn{5}{|l|}{ Time period } \\
\hline & 1988-1992 & 1993 & 1994-2001 & 2002 & $2003-2008$ \\
\hline Bird Island $\rightarrow$ Falkner Island & $0.004(0.001)$ & 0.000 & $0.002(0.001)$ & $0.003(0.002)$ & 0.000 \\
\hline Bird Island $\rightarrow$ Great Gull Island & $0.021(0.005)$ & 0.000 & $0.011(0.002)$ & $0.004(0.007)$ & $0.011(0.003)$ \\
\hline Bird Island $\rightarrow$ Penikese Island & - & - & - & $0.101(0.013)$ & $0.035(0.004)$ \\
\hline Bird Island $\rightarrow$ Ram Island & - & $0.009(0.004)$ & $0.211(0.014)$ & $0.114(0.021)$ & $0.145(0.008)$ \\
\hline Falkner Island $\rightarrow$ Bird Island & $0.015(0.004)$ & $0.078(0.018)$ & $0.010(0.003)$ & 0.000 & $0.012(0.007)$ \\
\hline Falkner Island $\rightarrow$ Great Gull Island & $0.077(0.012)$ & $0.047(0.017)$ & $0.080(0.010)$ & $0.097(0.040)$ & $0.062(0.021)$ \\
\hline Falkner Island $\rightarrow$ Penikese Island & - & - & - & $0.017(0.012)$ & 0.000 \\
\hline Falkner Island $\rightarrow$ Ram Island & - & $0.004(0.004)$ & $0.009(0.003)$ & 0.000 & $0.007(0.006)$ \\
\hline Great Gull Island $\rightarrow$ Bird Island & $0.037(0.005)$ & $0.064(0.013)$ & $0.014(0.002)$ & $0.011(0.006)$ & $0.007(0.002)$ \\
\hline Great Gull Island $\rightarrow$ Falkner Island & $0.022(0.004)$ & $0.026(0.007)$ & $0.016(0.002)$ & $0.004(0.004)$ & $0.006(0.002)$ \\
\hline Great Gull Island $\rightarrow$ Penikese Island & - & - & - & $0.016(0.006)$ & $0.008(0.002)$ \\
\hline Great Gull Island $\rightarrow$ Ram Island & - & $0.012(0.005)$ & $0.014(0.003)$ & $0.012(0.008)$ & $0.015(0.003)$ \\
\hline Penikese Island $\rightarrow$ Bird Island & - & - & - & - & $0.315(0.027)$ \\
\hline Penikese Island $\rightarrow$ Falkner Island & - & - & - & - & $0.007(0.005)$ \\
\hline Penikese Island $\rightarrow$ Great Gull Island & - & - & - & - & $0.026(0.011)$ \\
\hline Penikese Island $\rightarrow$ Ram Island & - & - & - & - & $0.384(0.030)$ \\
\hline Ram Island $\rightarrow$ Bird Island & - & - & $0.234(0.014)$ & $0.228(0.030)$ & $0.197(0.010)$ \\
\hline Ram Island $\rightarrow$ Falkner Island & - & - & $0.001(0.001)$ & 0.000 & $0.001(0.001)$ \\
\hline Ram Island $\rightarrow$ Great Gull Island & - & - & $0.018(0.004)$ & $0.004(0.004)$ & $0.013(0.003)$ \\
\hline Ram Island $\rightarrow$ Penikese Island & - & - & - & $0.100(0.015)$ & $0.029(0.004)$ \\
\hline
\end{tabular}

Source: Spendelow et al. (2010), Table 8 in Lebreton et al. (2003).

Table 10

Juvenile dispersal rates of Roseate Terns among the three major sites in the WWG, 1988-1997.

\begin{tabular}{llll}
\hline \multirow{2}{*}{ First breeding site } & \multicolumn{2}{l}{ Second breeding site } \\
\cline { 2 - 4 } & Falkner Island & Bird Island & Great Gull Island \\
\hline Falkner Island & $0.582^{*}$ & $0.004 \pm 0.004$ & $0.020 \pm 0.009$ \\
Bird Island & $0.047 \pm 0.060$ & $0.913^{*}$ & $0.082 \pm 0.038$ \\
Great Gull Island & $0.378 \pm 0.130$ & $0.082 \pm 0.042$ & $0.898^{*}$ \\
\hline
\end{tabular}

Source: Table 7 in Lebreton et al. (2003). Each entry is the estimate ( \pm standard error) of the probability that a bird raised as a chick at one site would breed for the first time at another site, conditional on survival to first breeding. Values on the diagonal $\left({ }^{*}\right)$ are fidelity rates ( 1 - sum of emigration rates); standard errors were not estimated for fidelity rates. Estimates are averaged over all years.

For each year $t$ and site $i$, a random number is drawn from a uniform distribution $u_{i, t} \sim U(0,1)$. If $N_{i, t}=0, \operatorname{decol}_{i, t}=0$ and $u_{i, t}<P\left(\operatorname{col}_{i}=1\right)$, small or medium-sized site $i$ enters colonization mode in year $t+1\left(\operatorname{col}_{i, t+1}=1\right)$. Likewise, if $N_{i, t}>0, \operatorname{col}_{i, t}=0$ and $u_{i, t}<P\left(\operatorname{decol}_{i}=1\right)$, small or medium-sized site $i$ enters decolonization mode in year $t+1\left(\right.$ decol $\left._{i, t+1}=1\right)$.

When a small or medium-sized site enters colonization mode, a number of pairs breed for the first time into the site. This number of pairs is drawn from a Poisson distribution $\left(N_{i, t+1} \sim \mathcal{P}(\lambda=\right.$ ExpSettlers)), where ExpSettlers is the expected number of pairs that colonize a previously abandoned site. This value has been estimated to 4 as the median from historical data (Table 4).

When a small or medium-sized site enters decolonization mode, a number of pairs leave the site. This number is drawn from a binomial distribution $\left(N_{i, t+1} \sim \mathcal{B}(\right.$ MaxDec, Pdec $\left.)\right)$, where MaxDec is the maximum number of pairs that have ever been observed in the historical data (U.S. Fish and Wildlife Service, 2010) to leave any site the year before that site was abandoned $N_{i, t+1}=0$, and Pdec is the probability that each pair leaves its current site in these events. Pdec is calculated as:

$\operatorname{Pdec}=\frac{\left.\sum_{j=1}^{M} \sum_{l=k_{1}}^{k_{T \max }} \operatorname{Pred}\left(N_{j, l}>0\right) \times \operatorname{Pred}\left(N_{j, l+1}=0\right) \times N_{j, l}\right)}{\operatorname{MaxDec} \times \sum_{j=1}^{M} \sum_{l=k_{1}}^{k_{T \max }} \operatorname{Pred}\left(N_{j, l}>0\right) \times \operatorname{Pred}\left(N_{j, l+1}=0\right)}$

These parameters are estimated from historical data (Table 5) as MaxDec $=37$ and $P d e c=0.026$. Once a small or medium-sized site has entered decolonization mode, it will remain in this mode until $N_{i, t}=0$, when decol $_{i, t}=0$. The model was implemented in R language (Core Team, 2016). Log-linear models were calculated using function $\mathrm{glm}$ from package stats, and the truncated normal distribution was simulated using package truncnorm (Trautmann et al., 2014).

Colonization and decolonization events override the dynamics defined by equation $N_{i, t+1}=\operatorname{round}\left(\left(A S_{i, t}+J S_{i, t}+I_{i, t+1}\right)\right)$ in the sites in colonization and decolonization mode. Moreover, the pairs that colonize any site the same year that it enters colonization mode (i.e., the settlers) are subtracted from the immigration pool in year $t$

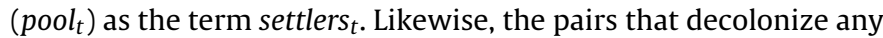
site the same year that it enters decolonization mode (i.e., the desettlers) are added to the immigration pool in year $t\left(\right.$ pool $\left._{t}\right)$ as the term desettlers $s_{t}$. These terms are calculated as settlers $s_{t}=\sum{ }_{i} N_{i, t+1}$ and desettlers $s_{t}=\sum_{j} N_{j, t}$, for each site $i$ and $j$ that enters colonization and decolonization mode, respectively, in year $t$. 
Colonizations per year

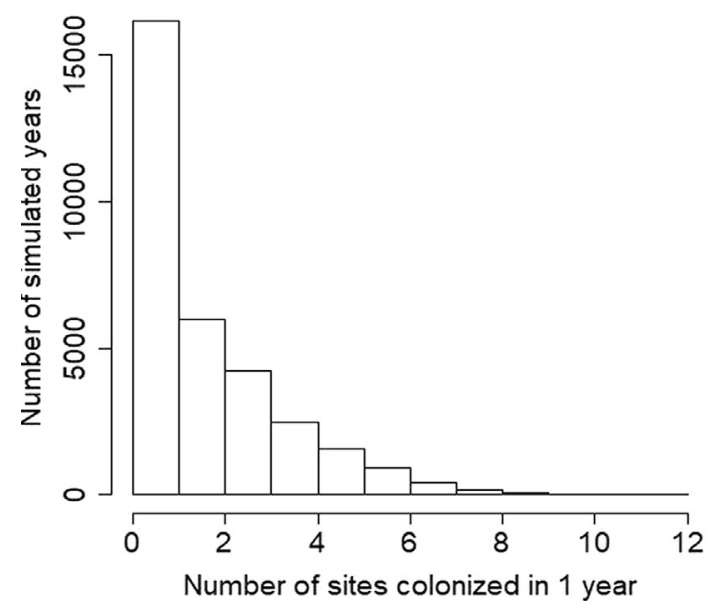

Decolonizations per year

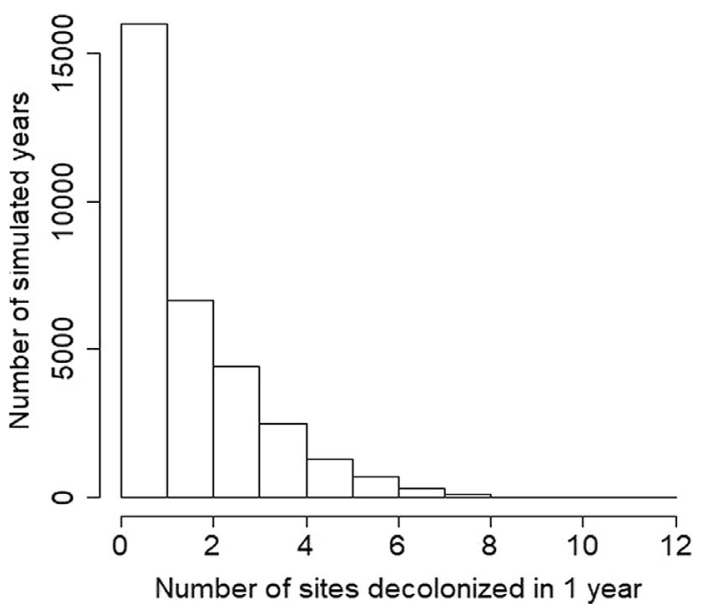

Fig. 2. Frequency distributions of colonizations (left) and decolonizations (right) among the 50,000 years simulated.

Number of pairs after colonization

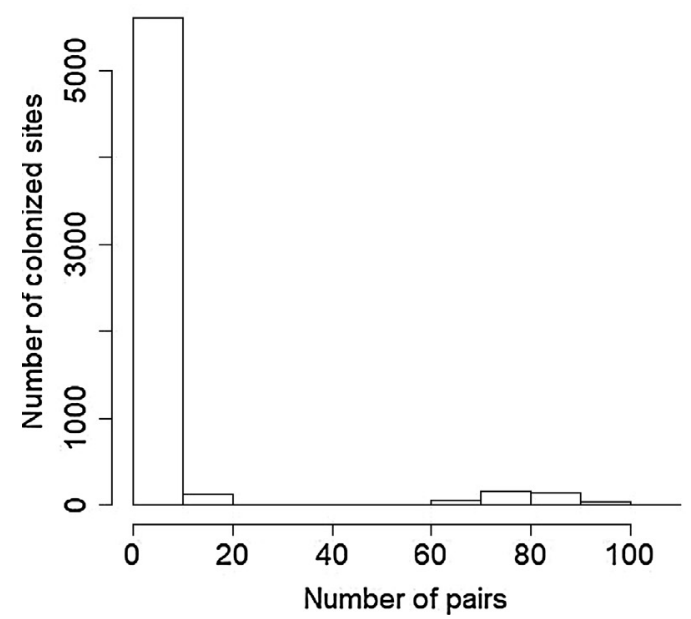

Number of pairs before decolonization

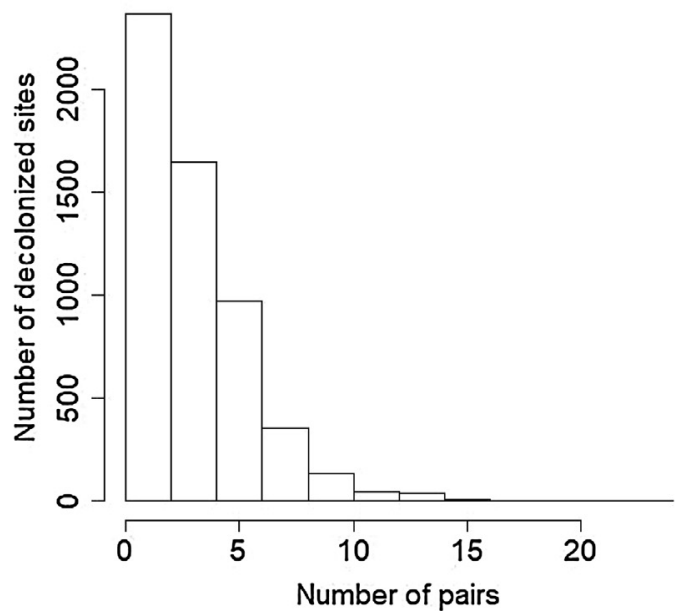

Fig. 3. Number of pairs that settle in a site in the year of its colonization (left) and number of pairs that abandon a site in the year of its decolonization (right).

\section{Results}

We ran 1000 simulations of our model; each run was simulated for 50 years. We analyzed the following metrics from the simulations' output: (a) the number of colonizations and decolonizations per year; (b) the number of pairs that colonize each site, i.e., breed at a site the year that it enters colonization mode; (c) the number of pairs that decolonize each site, i.e., abandon a site the year that it enters decolonization mode; (d) the proportion of birds that breed at 'large' sites at the end of each simulation; (e) the minimum and maximum number of pairs that breed in 'large' sites in each simulation across all simulated years; (f) the length of occupation, i.e., the number of years that 'small' and 'medium' colonies remain colonized (i.e., with at least 1 pair) until their abandonment; and $(\mathrm{g})$ the number of initially 'small' colonies that become 'large' or 'medium' during the simulated years and maintain this size until the end of the simulation. We also simulated one type of disaster by eliminating the breeders in a large colony.

The simulation results suggest that the percentage of years in which a single site was colonized is about $32 \%$ (around 16,000 from the total of $1000 \times 50=50,000$ years simulated). Years where 2 colonizations occur are more infrequent, and this tendency continues as the number of colonizations grows. This trend is mirrored by the distribution of decolonizations that occur in each year (Fig. 2).

The results also show that the majority of the colonizations and decolonizations involve a small number of pairs (1-10; Fig. 3). Nevertheless, a small proportion of colonizations involve a larger number of pairs, in the range $50-100$ pairs. These rare colonizations involving large numbers of pairs have been observed in historical data (Table 4). However, a few decolonizations involving 27-37 pairs (Table 5) were not captured by our model.

The model also predicts that the duration of occupation for 'small' and 'medium' size colonies is short in the majority of cases (the median and mode are 10 and 4 years respectively; Fig. 4). This result is consistent with the observed fact that most 'small' and 'medium' sites are intermittently occupied, i.e., they become colonized and then are abandoned a few years later (Table 2).

The simulations also predict that the proportion of pairs that breed in 'large' colonies in the last year of the simulation falls in the range $0.65-1.00$ (Fig. 5). This is a broader range than the proportion observed in the period 1988-2015 (0.85-0.99: Table 2), but proportions as low as 0.7 were recorded in historical data prior to 1988 (Nisbet, 1980).

The model predicts that the total number of pairs that breed in 'large' colonies is usually in the range $2700-4000$ (Fig. 6). Due to 


\section{Occupation of small and medium colonies}

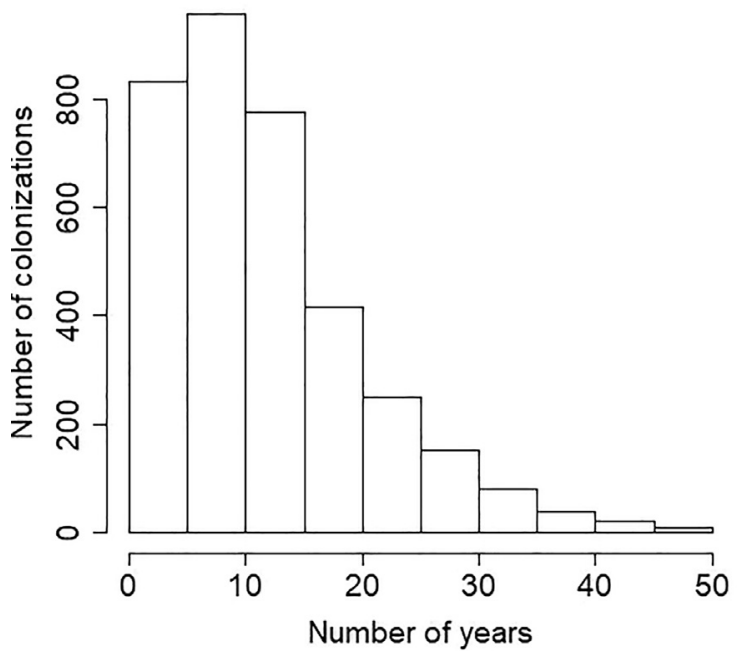

Fig. 4. Duration (years between colonization and decolonization) of occupation of 'small' and 'medium' colonies.

the unstable occupation dynamics of 'small' and 'medium' size sites, we decided to use this metric instead of using the total number of pairs across all sites. This metric is also consistent with historical data (Table 2).

The number of initially 'small' colonies that become 'medium' or 'large' and maintain this size until the last year of the 50-year simulation is about 1.2 and 1 , respectively. This conforms to the historical data, which indicate that the frequencies of formation of persistently 'large' and persistently 'medium' colonies are both on the order of $0.03 \mathrm{y}^{-1}$ (see Supplementary Material).

We also simulated an alternative scenario where one large colony (Bird Island) is not present in the system. This could emulate the effect of a sudden catastrophe in the population. With the exception of the pairs that were provided by this colony and are now absent, the metrics analyzed do not differ substantially from those from the original scenario. This could be an indicator of the high resilience of the studied system. In order to assess the sensitivity of our model to changes in parameters, we simulated different variations of it. Particularly, we analyzed the effect of changes in juvenile and adult fidelity on the proportion of birds that breed in

\section{Proportion of pairs in large colonies}

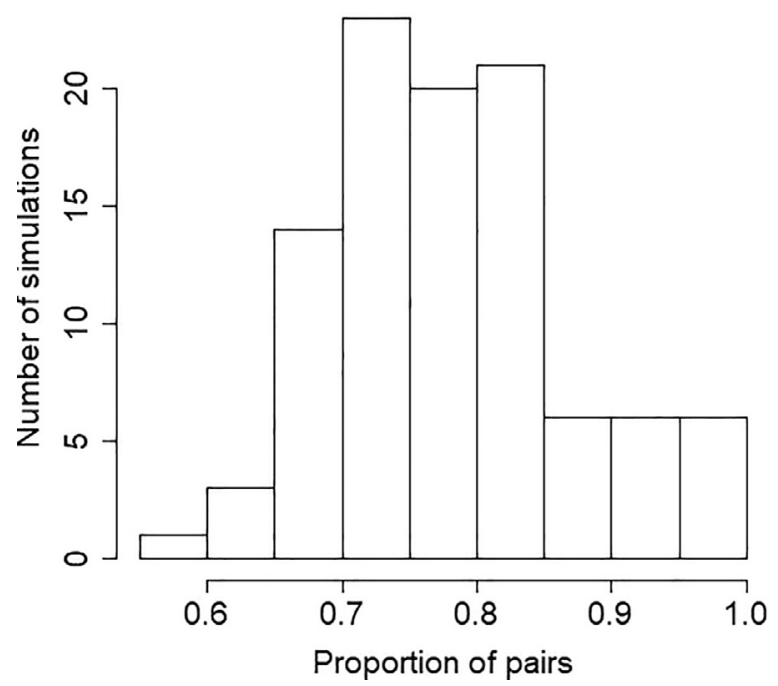

Fig. 5. Proportion of pairs that breed in 'large' colonies in the last year of each simulation.

'large' colonies in the last simulated year(Fig. 7). In these variations, a common (adult or juvenile) fidelity value was set for all colonies, overriding the derived values from the regression models for $\phi_{i, t}$ and $\rho_{i, t}$ defined in Section 2. The results show small fluctuations in the proportion of pairs breeding in 'large' colonies at the end of the simulation. However, we did not observe any clear tendency linking juvenile and adult fidelity with this proportion.

We also analyzed the effect of varying productivity, juvenile and adult survival on the maximum number of pairs in 'large' colonies across all simulated years (Fig. 8). The results show that the system collapses for values lower than a threshold. For instance, for adult survival this threshold is found around 0.8. After this threshold is reached, the results show an exponential relationship between each one of these parameters and this number of pairs. The steepness of this relationship depends on the altered parameter.

\section{Discussion and conclusions}

Seabird populations are often considered to function as metapopulations (e.g., Breton et al., 2014; Ponchon et al., 2015;

\section{Minimum pairs in large colonies}

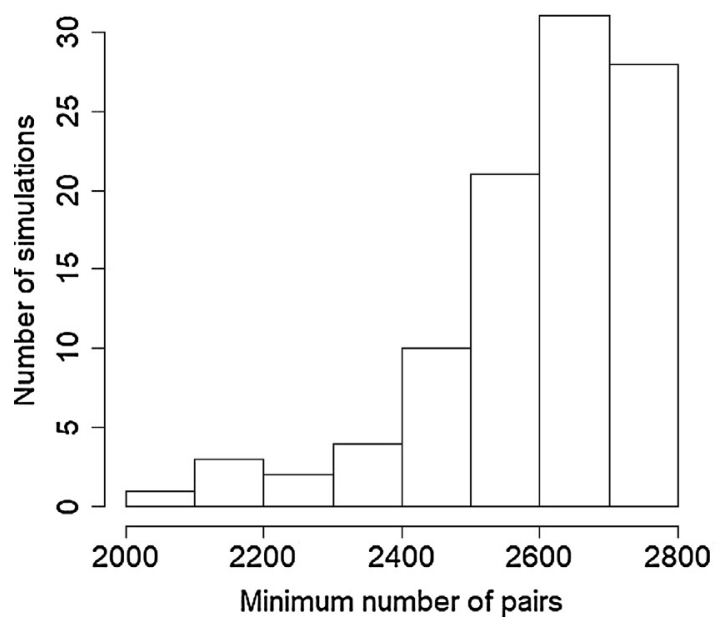

\section{Maximum pairs in large colonies}

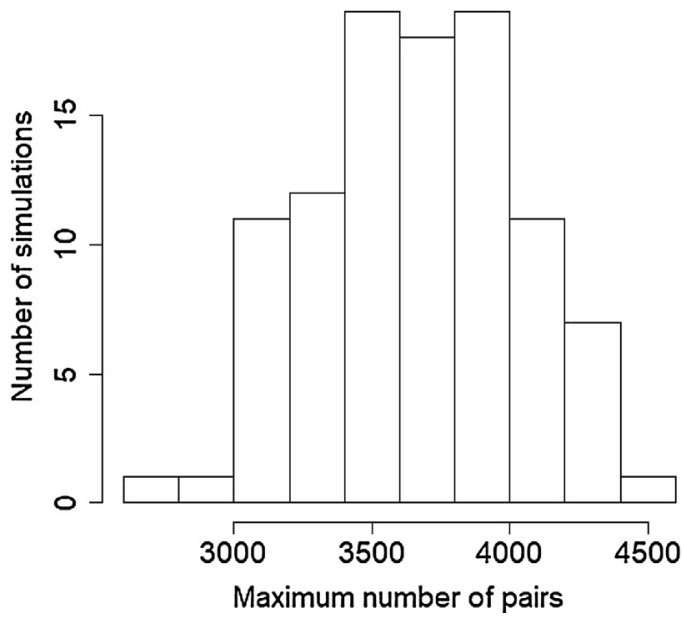

Fig. 6. Minimum (left) and maximum (right) number of pairs that breed in 'large' colonies in each simulation across all simulated years. 

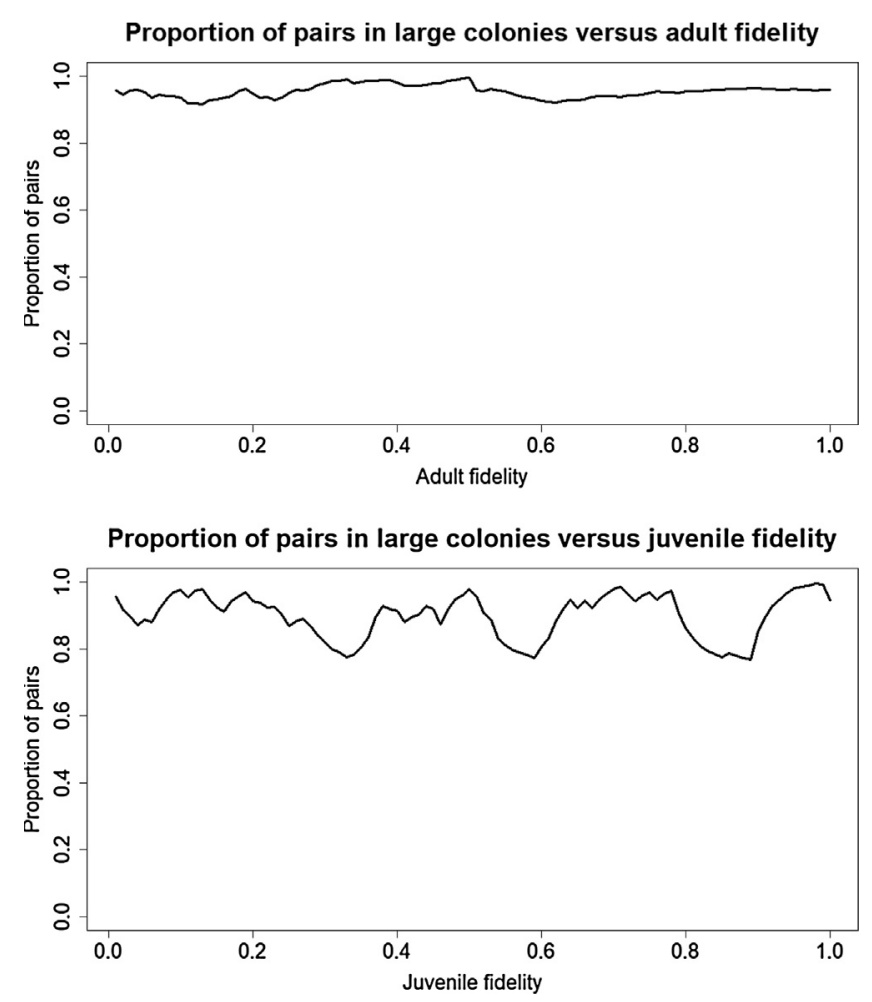

Fig. 7. Effect of changes in adult and juvenile fidelity on the proportion of pairs that breed in 'large' colonies in the last simulated year. The results show small oscillations in this proportion, but not a definite relationship.

Sanz-Aguilar et al., 2016). This tendency is driven, in part, by the naturally fragmented nature of the populations. This is insufficient, however, for concluding a metapopulation exists rather than a suite of isolated populations or a single large population (Smith and Green, 2005). One needs evidence of somewhat isolated local dynamics, as well as extinction-recolonization dynamics. Colonial seabirds can exhibit a type of metapopulation dynamics referred to as mainland-island metapopulation dynamics, whereby one or a few very large colonies exhibit long-term persistence, with ephemeral smaller patches that are colonized by birds emigrating from the larger patches (Dolman, 2012). Looking at the map (Fig. 1), three large colonies lie on an axis of about $140 \mathrm{~km}$ running from WSW to ENE, and almost all of the 'small' and 'medium' colonies lie either to the west or east of them. This is probably a quirk of geomorphology; there are very few suitable islands along this axis except for the three occupied by large colonies (historically, Penikese Island was also very large, but that conforms to the same pattern). Thus, the mainland-island configuration of our metapopulation is also a central-peripheral pattern.

The biggest difficulty in understanding metapopulation dynamics for seabirds can be documenting, quantifying, and modeling colonization and decolonization events (Payo-Payo et al., 2017; Sherley et al., 2017; Southwell et al., 2017). This can be problematic when colonization events are rare (Munilla et al., 2016), which can occur when most colonies are large and in species where social dynamics affect colonization (Ponchon et al., 2015; Reed and Levine, 2005; Schippers et al., 2011). The difficulty of understanding colonization-decolonization dynamics is exacerbated in species with delayed breeding, by the difficulty of tracking juvenile and long-distance movements (Bicknell et al., 2014; Yannic et al., 2016). We used data from a long-term study to quantify the long-term dynamics of site occupancy of roseate terns in the north Atlantic (Spendelow et al., 2016), and we used the model to make inferences about the dynamics governing the system.
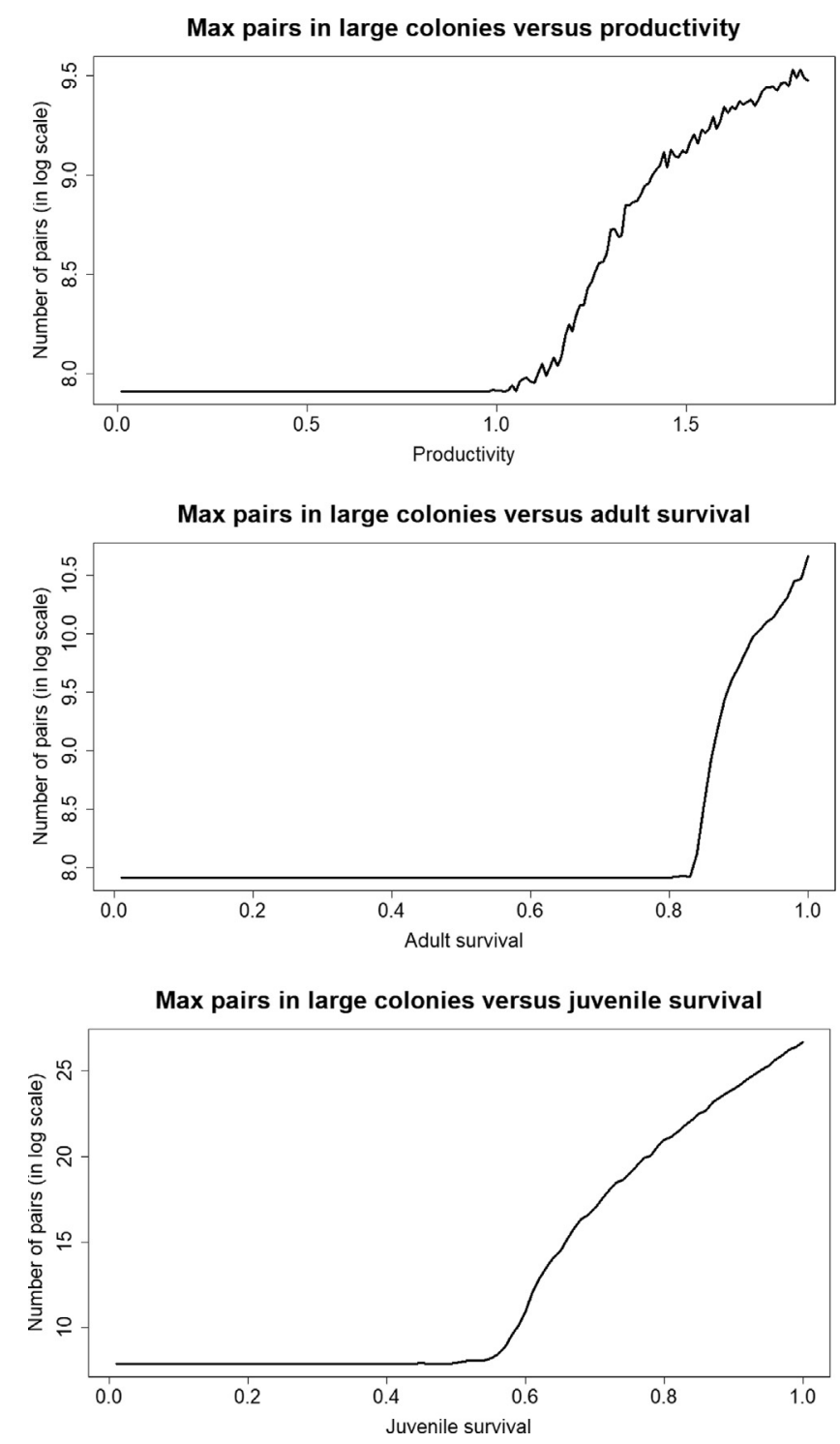

Fig. 8. Effect of changes in different parameters on the maximum number of pairs in 'large' colonies across all simulated years. These numbers of pairs are shown in logarithmic scale.

In our system, productivity increases strongly with site quality $\left(q_{i}\right)$ which is destabilizing in the sense that it would increase the concentration of the population into a few large colonies with high $q_{i}$. Any additional dependence on $N_{i, t}$ would be additionally destabilizing in the sense that it could lead to a runaway increase in the total population. Feeding into this cycle might be conspecific attraction of dispersers, which is often exhibited by colonial species (Reed and Dobson, 1993). Counteracting this destabilizing tendency, we included an inverse relationship between juvenile survival and site quality (where quality was defined as average population size). So, at our larger sites, we modeled, and find field evidence, of density dependence acting in the large colonies. There is some evidence of density dependent colonization dynamics reported in other seabird metapopulations (Lisnizer et al., 2015; Matthiopoulos et al., 2005; Pozzi et al., 2015), as well as source-sink dynamics (Lisnizer et al., 2015; Ponchon et al., 2015; Sanz-Aguilar et al., 2016). As might be expected in a mainland-island metapopulation, we found different local dynamics in 'small' and 'medium' colonies in our system. These exhibited dynamics that were more stochastic and density independent than those of the 'large' colonies, resulting in local decolonizations and recolonizations. We found that we had to 
model colonization and decolonization events separately from our general model of emigration from sites and allocation of the pool of immigrants. When we did not model colonization and decolonization as special cases, our model predicted that these events usually involve large numbers of pairs spearheading the colonization and abandonment of sites. Although colonizations and decolonizations involving large numbers of pairs exist in the historical data, they are rare, and the vast majority of colonizations and decolonizations involve small numbers of pairs. Therefore, we had to adapt the model so that it reflects this property of the system. The difference in local dynamics between 'small' and 'medium' sites and 'large' sites reinforces our view that 'small' and 'medium' sites were qualitatively different from large sites - consequently, the processes governing their dynamics were modeled differently.

In our model, productivity, adult survival and juvenile survival appear to be the fundamental aspects that make the model consistent with the observations. In other words, minor changes in these aspects have profound effects on the dynamics of the model, and slight deviations in the parameter values obtained from our data rapidly lead the model dynamics either to population collapse or to uncontrolled growth in the numbers of pairs, resulting in levels of population significantly larger than in the observed data. We also found that expected population size of large colonies exhibits a threshold sensitivity to adult survival. That is, over a very small range of values, populations went from large to non-existent. This type of threshold vulnerability to changes in adult survival has been reported before (Mortensen and Reed, 2016), but it is not often investigated. Traditional vital rate sensitivity analyses sometimes report adult survival rate to be the most sensitive vital rate for population growth rate in species with low reproductive rates (Breininger et al., 1999), but there is little consistency in this result across analytical methods (Fefferman and Reed, 2006; Mortensen and Reed, 2016; Wisdom et al., 2000).

Conversely, variations in fidelity do not seem to have a significant impact on the dynamics of the model. The model simulations reflect a strong dichotomic distribution with 'large' colonies on one side and 'small' and 'medium' colonies on the other. The vast majority of the pairs (about $80-90 \%$ ) breed in 'large' colonies during the last year of each simulation. Changes in the parameters associated with fidelity cause minor oscillations on these percentages, but do not reflect a clear monotonic trend linking fidelity and proportion of pairs that breed in 'large' colonies. Interestingly, we found a small but unexpected oscillatory relationship between juvenile and adult fidelity and the proportion of pairs in 'large' colonies in the last year of each simulation. This relationship is especially clear for juvenile fidelity. We were unable to give an explanation for this phenomenon, and we appreciate any hypotheses from readers that would shed light on it.

The inverse correlation between juvenile survival and site quality acts as a stabilizing factor of the population and counterbalances the destabilizing effect of the positive correlation between productivity and site quality. This latter positive correlation tends to destabilize the system because it creates a positive feedback between site quality and productivity that causes the population to grow unrestrained.

Our model treats dispersal as a two-step process. Emigration from colony site $i$ is treated as an intrinsic property of site $i$, and is independent of the destination colonies of the emigrating pairs. Pairs that emigrate from all sites enter a common pool, and are redistributed with the condition that they do not breed in the same colony from which they emigrated in the same year. The proportion of pairs that immigrate into colony site $j \neq i$ is modeled as a function of $q_{j}$, and is independent of the colonies of origin of the immigrating pairs. We found it unnecessary to explicitly consider pairwise co-dependences to model migration between each pair of sites.
We also found that colonization and decolonization needed to be modeled as separate events from the rest of the migration dynamics. This was because the colonization and decolonization dynamics of 'small' and 'medium' colonies and 'large' colonies are different. In fact, colonization and decolonization dynamics of 'small' and 'medium' colonies are stochastic events that frequently involve large numbers of pairs and apparently do not occur in 'large' colonies.

Our model successfully reproduces several distinctive features of the Northwest Atlantic metapopulation of roseate terns:

- The metapopulation includes 2-4 'large' colonies which hold $85-95 \%$ of the total numbers and remain similarly 'large' for long periods. In our model, this results from our assumptions that each colony site is characterized by a single constant (intrinsic 'quality'), that adult and juvenile fidelity rates are directly correlated with quality, and that birds that leave their origin sites are allocated among possible destination sites in proportion to the relative quality of these destination sites, generating a sourcesink dynamics. We estimated the quality of each site from the average number of pairs over the 28-year observation period.

- The metapopulation also includes about 30 sites with low or medium values of intrinsic quality, most of which are occupied intermittently; colonizations and decolonizations are fairly frequent and sometimes involve much larger numbers of birds than what the intrinsic quality of the site would indicate. In our model, we found it necessary to model colonizations and decolonizations as stochastic events separately from our model of dispersal among sites already occupied, which predicted only small numbers of birds entering or leaving low-quality sites.

- The numbers of breeding pairs in each of the 'large' colonies and the total number of pairs in the metapopulation remain within moderately narrow bounds over periods of decades, in spite of positive correlations between colony size and breeding productivity, adult fidelity and juvenile fidelity, all of which would be expected to be destabilizing. In our model, both colony and total numbers are stabilized by introducing an inverse relationship between juvenile survival and colony size. We did not find it necessary to include other negatively density-dependent factors, either at the level of the colony or the metapopulation.

- In addition to colonizations and decolonizations, numbers of pairs at the 'large' and 'medium' colonies fluctuate from year to year, and several of the demographic parameters that have been measured (juvenile survival, adult and juvenile dispersal rates) also vary among years. Our model was able to reproduce the observed fluctuations in numbers by incorporating stochastic variation in the same demographic parameters, being the amplitude of this variation adjusted to approximate the observed range.

- In the variations of the model where common values for adult survival, juvenile survival and productivity rates were used for all years and sites, there exists a tipping point for the value of adult survival rate around 0.835 over which the population in the system becomes unstable in the long term (Fig. 8). This is consistent with the data from field observations (Table 6). The tipping point for productivity in our model (approximately 1.1) is also consistent with field data (Table 3 ). The sensitivity of our model to changes in the value of these parameters may help explain the observed oscillations in our population.

\section{Acknowledgements}

JMR was partly supported by a Charles Bullard Fellowship in Forest Research from Harvard Forest, Harvard University. MGQ was partly supported by a Smithsonian Fellowship from Smithsonian Institution. The authors also acknowledge all colony managers for 
data, particularly H. Hays and J. Spendelow, and the New Bedford Harbor Trustee Council, which supported the work in Buzzards Bay, MA.

\section{Appendix A. Supplementary data}

Supplementary data associated with this article can be found, in the online version, at https://doi.org/10.1016/j.ecolmodel.2017.12. 007.

\section{References}

Bicknell, A.W.J., Knight, M.E., Bilton, D.T., Campbell, M., Reid, J.B., Newton, J., Votier, S.C., 2014. Intercolony movement of pre-breeding seabirds over oceanic scales: implications of cryptic age-classes for conservation and metapopulation dynamics. Divers. Distrib. 20, 160-168, http://dx.doi.org/10.1111/ddi.12137.

Breininger, D.R., Burgman, M.A., Stith, B.M., 1999. Influence of habitat quality, catastrophes, and population size on extinction risk of the Florida scrub-jay. Wildl. Soc. Bull. (1973-2006) 27, 810-822 http://www.jstor.org/stable/ 3784104.

Breton, A.R., Nisbet, I.C.T., Mostello, C.S., Hatch, J.J., 2014. Age-dependent breeding dispersal and adult survival within a metapopulation of common terns (Sterna hirundo). Ibis 156, 534-547, http://dx doi.org/10.1111/ibi.12161.

Crespin, L., Harris, M.P., Lebreton, J.D., Frederiksen, M., Wanless, S., 2006. Recruitment to a seabird population depends on environmental factors and on population size. J. Anim. Ecol. 75, 228-238, http://dx.doi.org/10.1111/j.13652656.2006.01035.x.

Dolman, P.M., 2012. Mechanisms and processes underlying landscape structure effects on bird populations. In: Fuller, R.J. (Ed.), Birds and Habitat. Cambridge University Press, Cambridge, pp. 93-124, http://dx.doi.org/10.1017/ CB09781139021654.006 http://ebooks.cambridge.org/ref/id/ CB09781139021654A011.

Fefferman, N.H., Reed, J.M., 2006. A vital rate sensitivity analysis for nonstable age distributions and short-term planning. J. Wildl. Manag. 70, 649-656, http://dx. doi.org/10.2193/0022-541X(2006)70[649:AVRSAF]2.0.CO;2.

Hanski, I., 2001. Population dynamics consequences of dispersal in local populations and in metapopulations. In: Clobert, J., Danchin, E., Dhondt, A.A., Nichols, J.D. (Eds.), Dispersal. Oxford University Press, New York, pp. 283-298.

Harrison, S., Taylor, A.D., 1997. Empirical evidence for metapopulation dynamics. In: Hanski, I., Gilpin, M.E. (Eds.), Metapopulation Biology: Ecology, Genetics, and Evolution. University of Minnesota Press, Minneapolis, MN, USA, pp. $27-42$

Jønsson, K.A., Tøttrup, A.P., Borregaard, M.K., Keith, S.A., Rahbek, C., Thorup, K., 2016. Tracking animal dispersal: From individual movement to community assembly and global range dynamics. Trends Ecol. Evol. 31, 204-214, http://dx. doi.org/10.1016/j.tree.2016.01.003.

Koenig, W.D., Van Vuren, D., Hooge, P.N., 1996. Detectability, philopatry, and the distribution of dispersal distances in vertebrates. Trends Ecol. Evol. 11, 514-517, http://dx.doi.org/10.1016/S0169-5347(96)20074-6.

Lebreton, J.D., Hines, J.E., Pradel, R., Nichols, J.D., Spendelow, J.A., 2003. Estimation by capture-recapture of recruitment and dispersal over several sites. Oikos 101, 253-264, http://dx.doi.org/10.1034/j.1600-0706.2003.11848.x.

Lisnizer, N., García-Borboroglu, P., Pascual, M., Yorio, P., 2015. Transfer processes drive population dynamics of kelp gull colonies in Patagonia: implications for management strategies. Mar. Biol. Res. 11, 738-746, http://dx.doi.org/10.1080/ 17451000.2014.993652.

Matthiopoulos, J., Harwood, J., Thomas, L., 2005. Metapopulation consequences of site fidelity for colonially breeding mammals and birds. J. Anim. Ecol. 74, 716-727, http://dx.doi.org/10.1111/j.1365-2656.2005.00970.x.

Mortensen, J.L., Reed, J.M., 2016. Population viability and vital rate sensitivity of an endangered avian cooperative breeder, the white-breasted thrasher (Ramphocinclus brachyurus). PLOS ONE 11, e0148928, http://dx.doi.org/10. 1371/journal.pone.0148928.

Munilla, I., Genovart, M., Paiva, V.H., Velando, A., Baudouin, L., Estoup, A., Ropert-Coudet, Y., Forero, M., 2016. Colony foundation in an oceanic seabird. PLOS ONE 11, e0147222, http://dx.doi.org/10.1371/journal.pone.0147222.

Nisbet, I.C.T., 1980. Status and trends of the roseate tern Sterna dougallii in North America and the Caribbean, Report to U.S. Fish \& Wildlife Service, Office of Endangered Species.

Nisbet, I.C.T., 2014. Roseate Tern Sterna dougallii. The Birds of North America Online, No. 370 http://birdsna-org.bnaproxy.birds.cornell.edu/SpeciesAccount/bna/species/roster/.

Nisbet, I.C.T., Monticelli, D., Spendelow, J.A., Szczys, P., 2016. Prebreeding survival of roseate terns Sterna dougallii varies with sex, hatching order and hatching date. Ibis 158, 327-334, http://dx.doi.org/10.1111/ibi.12359.

Opdam, P., 1991. Metapopulation theory and habitat fragmentation: a review of Holarctic breeding bird studies. Landsc. Ecol. 5, 93-106, http://dx.doi.org/10. 1007/BF00124663.
Payo-Payo, A., Genovart, M., Sanz-Aguilar, A., Gre no, J.L., García-Tarrasón, M., Bertolero, A., Piccardo, J., Oro, D., 2017. Colonisation in social species: the importance of breeding experience for dispersal in overcoming information barriers. Sci. Rep. 7, 42866, http://dx.doi.org/10.1038/srep42866.

Ponchon, A., Garnier, R., Grémillet, D., Boulinier, T., 2015. Predicting population responses to environmental change: the importance of considering informed dispersal strategies in spatially structured population models. Divers. Distrib. 21, 88-100, http://dx.doi.org/10.1111/ddi.12273.

Pozzi, L.M., Borboroglu, P.G., Boersma, P.D., Pascual, M.A., Gallelli, H., Upton, J., 2015. Population regulation in Magellanic penguins: what determines changes in colony size? PLOS ONE 10, e0119002, http://dx.doi.org/10.1371/journal. pone.0119002.

Core Team, R., 2016. R: A Language and Environment for Statistical Computing. R Foundation for Statistical Computing, Vienna, Austria https://www.R-project. org/.

Reed, J., Dobson, A.P., 1993. Behavioural constraints and conservation biology: conspecific attraction and recruitment. Trends Ecol. Evol. 8, 253-256, http:// dx.doi.org/10.1016/0169-5347(93)90201-Y.

Reed, J.M., Levine, S.H., 2005. A model for behavioral regulation of metapopulation dynamics. Ecol. Model. 183, 411-423, http://dx.doi.org/10.1016/j.ecolmodel. 2004.02.025.

Rhodes, O.E., Chesser, R.K., Smith, M.H., 1996. Population Dynamics in Ecological Space and Time. University of Chicago Press, Chicago.

Sanz-Aguilar, A., Igual, J.M., Tavecchia, G., Genovart, M., Oro, D., 2016. When immigration masks threats: the rescue effect of a Scopoli's shearwater colony in the Western Mediterranean as a case study. Biol. Conserv. 198, 33-36, http://dx.doi.org/10.1016/j.biocon.2016.03.034.

Saura, S., Bodin, Ö., Fortin, M.J., 2014. Stepping stones are crucial for species' long-distance dispersal and range expansion through habitat networks. J. Appl. Ecol. 51, 171-182, 10.1111/1365-2664.12179.

Schippers, P., Stienen, E.W., Schotman, A.G., Snep, R.P., Slim, P.A., 2011. The consequences of being colonial: allee effects in metapopulations of seabirds. Ecol. Model. 222, 3061-3070, http://dx.doi.org/10.1016/j.ecolmodel.2011.05. 022.

Serrano, D., Oro, D., Ursúa, E., Tella, J., 2005. Colony size selection determines adult survival and dispersal preferences: allee effects in a colonial bird. Am. Nat. 166, E22-E31, http://dx.doi.org/10.1086/431255.

Sherley, R.B., Ludynia, K., Dyer, B.M., Lamont, T., Makhado, A.B., Roux, J.P., Scales, K.L., Underhill, L.G., Votier, S.C., Chivers, L., et al., 2017. Metapopulation tracking juvenile penguins reveals an ecosystem-wide ecological trap. Curr. Biol. 27, 563-568, http://dx.doi.org/10.1016/j.cub.2016.12.054.

Smith, A.M., Green, D.M., 2005. Dispersal and the metapopulation paradigm in amphibian ecology and conservation: are all amphibian populations metapopulations? Ecography 28, 110-128, http://dx.doi.org/10.1111/j.09067590.2005.04042.x.

Southwell, C., Emmerson, L., Takahashi, A., Kato, A., Barbraud, C., Delord, K., Weimerskirch, H., 2017. Recent studies overestimate colonization and extinction events for Adelie penguin breeding colonies. Auk 134, 39-50, http:// dx.doi.org/10.1642/AUK-16-125.1.

Spendelow, J., Hines, J., Nichols, J., Nisbet, I., Cormons, G., Hays, H., Hatch, J., Mostello, C., 2008. Temporal variation in adult survival rates of roseate terns during periods of increasing and declining populations. Waterbirds 31 , 309-319.

Spendelow, J.A., Monticelli, D., Nichols, J.D., Hines, J.E., Nisbet, I.C.T., Cormons, G., Hays, H., Hatch, J.J., Mostello, C.S., 2016. Roseate tern breeding dispersal and fidelity: responses to two newly restored colony sites. Ecosphere 7, e01510, http://dx.doi.org/10.1002/ecs2.1510.

Spendelow, J.A., Mostello, C.S., Nisbet, I.C.T., Hall, S.S., Welch, L., 2010. Interregional breeding dispersal of adult roseate terns. Waterbirds 33, 242-245, http://dx. doi.org/10.2307/1521572.

Stith, B.M., Fitzpatrick, J.W., Woolfenden, G.E., Pranty, B., 1996. Classification and conservation of metapopulations: a case study of the Florida scrub jay. In: McCullough, D.R. (Ed.), Metapopulations and Wildlife Conservation. Island Press, Washington, DC, USA, pp. 187-215.

Trautmann, H., Steuer, D., Mersmann, O., Bornkamp, B., 2014. truncnorm: Truncated normal Distribution, r package version 1.0-7 https://CRAN.R project.org/package=truncnorm.

U.S. Fish and Wildlife Service, 2010. Caribbean Roseate Tern and North Atlantic Roseate Tern (Sterna dougallii). 5-year review: Summary and evaluation. U.S. Fish \& Wildlife Service, Boquerón, Puerto Rico, and Concord, New Hampshire.

Whitlock, M.C., 2001. Dispersal and the genetic properties of metapopulations. In: Clobert, J., Danchin, E., Dhondt, A.A., Nichols, J.D. (Eds.), Dispersal. Oxford University Press, New York, pp. 273-282.

Wisdom, M.J., Mills, L.S., Doak, D.F., 2000. Life stage simulation analysis: estimating vital-rate effects on population growth for conservation. Ecology 81, 628-641 http://www.jstor.org/stable/177365.

Yannic, G., Yearsley, J.M., Sermier, R., Dufresnes, C., Gilg, O., Aebischer, A., Gavrilo, M.V., Strøm, H., Mallory, M.L., Morrison, R.I.G., Gilchrist, H.G., Broquet, T., 2016 High connectivity in a long-lived high-Arctic seabird, the ivory gull Pagophila eburnea. Polar Biol. 39, 221-236, http://dx.doi.org/10.1007/s00300-015-1775z. 